\title{
Naumann, Johannes
}

\section{A model of online reading engagement. Linking engagement, navigation, and performance in digital reading}

formal und inhaltlich überarbeitete Version der Originalveröffentlichung in:

formally and content revised edition of the original source in:

Computers in human behavior 53 (2015), S. 263-277

Bitte verwenden Sie in der Quellenangabe folgende URN oder DOI /

Please use the following URN or DOI for reference:

urn:nbn:de:0111-pedocs-179745

10.25656/01:17974

https://nbn-resolving.org/urn:nbn:de:0111-pedocs-179745

https://doi.org/10.25656/01:17974

\section{Nutzungsbedingungen}

Dieses Dokument steht unter folgender Creative Commons-Lizenz: http://creativecommons.org/licenses/by-nc-nd/4.0/deed.de - Sie dürfen das Werk bzw. den Inhalt unter folgenden Bedingungen vervielfältigen, verbreiten und öffentlich zugänglich machen: Sie müssen den Namen des Autors/Rechteinhabers in der von ihm festgelegten Weise nennen. Dieses Werk bzw. dieser Inhalt darf nicht für kommerzielle Zwecke verwendet werden und es darf nicht bearbeitet, abgewandelt oder in anderer Weise verändert werden.

Mit der Verwendung dieses Dokuments erkennen Sie die Nutzungsbedingungen an.

\section{Terms of use}

This document is published under following Creative Commons-License: http://creativecommons.org/licenses/by-nc-nd/4.0/deed.en - You may copy, distribute and transmit, adapt or exhibit the work in the public as long as you attribute the work in the manner specified by the author or licensor. You are not allowed to make commercial use of the work or its contents. You are not allowed to alter, transform, or change this work in any other way.

By using this particular document, you accept the above-stated conditions of use.

\section{Kontakt / Contact:}

peDOCS

DIPF | Leibniz-Institut für Bildungsforschung und Bildungsinformation Informationszentrum (IZ) Bildung

E-Mail: pedocs@dipf.de

Internet: www.pedocs.de 
Running head: Online reading engagement

A model of online reading engagement: Linking engagement, navigation, and performance in digital reading

Johannes Naumann

German Institute for International Educational Research (DIPF) and Goethe University

Frankfurt am Main, Germany

Manuscript accepted for publication in the Journal Computers in Human Behavior

Author note. Johannes Naumann is now at Goethe-University, Frankfurt am Main, Germany, and an associated scientist with DIPF. Parts of the present research emerged while he worked at DIPF, while other parts emerged while he was at Goethe-University. Please address correspondence concerning this article to

Johannes Naumann

Goethe-Universität

Senckenberganlage 31

60325 Frankfurt am Main, Germany

Tel. $++49(0) 6979823001$

E-Mail j.naumann@em.uni-frankfurt.de 


\begin{abstract}
A model of online reading engagement is outlined. This model proposes that online reading engagement predicts dedication in digital reading. Dedication in digital reading according to the model is reflected in task-adaptive navigation, and task-adaptive navigation predicts digital reading performance over and above print reading skill. Information engagement is assumed to positively predict task-adaptive navigation, while social engagement is assumed to negatively predict task-adaptive navigation. These hypotheses were tested using OECD PISA
\end{abstract} 2009 Digital Reading Assessment data from 17 countries and economies $(\mathrm{N}=29,395)$. Individual task responses served as the primary unit of analysis. Linear mixed models were used to predict navigation behavior from the interaction of information and social online reading engagement with navigation demands. High information engagement was associated with more task-adaptive navigation behavior, as shown by significant positive interactions between information engagement and tasks' navigation demands. In contrast, high social engagement was associated with less adaptive navigation behavior, as shown by negative interactions between social engagement and navigation demands. Generalized linear mixed models were used to predict task performance by the interaction of navigation demands and navigation behavior. Adaptive navigation behavior predicted digital reading task performance, as shown by significant interactions between navigation behavior and navigation demands. These results are in support of the proposed model of online reading engagement. Keywords: hypertext; navigation; reading engagement; digital reading; PISA; log file 


\section{Introduction}

The aim of the present article is to outline a model of online reading engagement, and put forward a test of two crucial parts of this model. In a nutshell, this model assumes that long-term online reading habits (online reading engagement) predict task-adaptive navigation. Task-adaptive navigation means navigation behavior that is responsive to task demands, as information is accessed and processed as required by the task, while task-irrelevant information is discarded. Different effects are assumed for online reading engagement targeted at information seeking (information engagement), versus online reading engagement targeted at social interaction (social engagement). While information engagement is assumed to positively predict task-adaptive navigation, social engagement is expected to negatively predict task adaptive navigation Task-adaptive navigation according to the model predicts digital reading performance over and above print reading skill. These assumptions were tested in 17 samples of 15 -year olds.

\subsection{Reading engagement}

As of 2014, over 2.4 billion people have access to the Internet worldwide (Miniwatts Marketing Group, 2012). The Internet has grown a major resource for dissemination of news, opinion, and knowledge. A person who for whatever reason is unable to access these resources will be cut off major streams of societal and political debate. Being in command of online reading skill is crucial for participation in $21^{\text {st }}$ century societies, both in private and occupational contexts. For example, online reading skill will be required to successfully employ ICTs in daily (e.g. OECD, 2013), or occupational life (e.g. when engaging in the process of knowledge sharing using ICTs in the workplace, see Zhan, de Pablos, \& Zhu, 2013 and Zhang, Vogel, \& Zhu, 2012). One predictor of reading skill in general is reading engagement (Guthrie, Wigfield \& You, 2012). Reading engagement is specifically shaped in online reading, as online reading cannot perfectly be mapped upon print reading (e.g. Leu, Kinzer, Coiro, and Cammack, 2004; Rouet, 2006). Engaged reading is motivated, strategic, 
knowledge-driven, and socially interactive (Guthrie et al., 2004; Guthrie et al., 2012).

Motivated means that readers do not only read for external gains, but are curious for a text's contents, or enjoy reading itself. Strategic means that they employ cognitive and metacognitive strategies such as summarizing, or monitoring comprehension. Knowledgedriven means that readers use their prior knowledge to better understand a text, and use the text to increase their knowledge. Socially interactive means that readers engage with significant others in debating a text's stance, or to share their reading experiences. Guthrie et al. (2012) proposed a framework in which these components of reading engagement are put into a causal order. In this framework, classroom practice and conditions impact motivations to read, which in turn impact behavioral engagement and dedication in reading. This latter processes, behavioral engagement and dedication, is what ultimately predicts reading competence (see also Wigfield et al., 2008).

\subsection{Model of online reading engagement}

The model of online reading engagement is depicted in Figure 1. While the model shares a number of assumptions with Guthrie et al.'s (2012) model of general reading engagement, it also makes some additional assumptions. Most importantly, it discriminates between a student level and a task level. Through this, the effects of student level variables, the effects of task level variables and their interactions can explicitly be modeled.

1.2.1 Prediction of digital reading performance. The model seeks at predicting performance in digital reading tasks, a variable that is located at the task level. First, performance is predicted by a student's print reading skills (path p1 in Figure 1). This is because reading digital text is reading in the first place, and thus skills involved in reading printed text such as word decoding, sentence integration, and situation model formation will impact performance in reading digital text as well. Second, digital reading task performance is predicted by the task, and a student's engagement with that task. In reading digital text, one important part of task engagement is "navigation" (see Lawless \& Schrader, 2008, for an in- 
depth discussion of the 'navigation' metaphor). Digital texts frequently come as hypertexts (e.g. Afflerbach \& Cho, 2008). This means that the text is not being read linearly from beginning to end, but the contents is distributed across a number distinct pages, or 'nodes', which are interconnected through hyperlinks. It is thus left to the reader to find an appropriate selection of text materials. This is even more so as reading digital text typically not only requires selecting relevant text materials, but also not getting distracted by non-relevant materials, be it parts of the text, or accompanying media such as movies, animations, or photos. Previous research has shown that students who get distracted by task-irrelevant contents ("feature explorers", Lawless \& Kulikowich, 1996) achieve less in comprehension (see also Salmerón, Kintsch, \& Cañas, 2006). These findings are very well aligned with Guthrie et al.'s (2012) general description of behavioral engagement in reading, who coin behavioral engagement in reading 'dedication' (p. 604, Fig. 29.1). It is those students who do a good job in selecting text contents (e.g. Cress \& Knabel, 2003; Hsu \& Schwen, 2003; Naumann, Richter, Christmann \& Groeben, 2008; Puntambekar, Stylianou, \& Hübscher, 2003; Richter, Naumann \& Noller, 2003) and organizing text contents (e.g. Amadieu, Tricot, \& Mariné, 2009; Salmerón, Cañas, Kintsch, \& Fajardo, 2005; Su \& Klein, 2006; Puntambekar \& Stylianou, 2005; Richter, Naumann, Brunner \& Christmann, 2005) who perform well in digital reading. Digital reading performance is thus expected to be predicted by navigation (path p2 in Figure 1).

Navigation behavior is a broad construct, and can be measured by a multitude of indicators. These may e.g. encompass the linearity or connectedness of a navigational path (e.g. McEneaney, 2001; Richter et al., 2003), the semantic coherence of a navigational path (e.g. Salmerón et al., 2005), or the attendance to task-relevant pages (e.g. Naumann et al., 2008), or the number of page visits overall (e.g. Su \& Klein, 2006). How one particular instance of navigation behavior, e.g. the number of page visits, will be associated with digital reading performance is moderated by task demands. Consider integrating information from 
multiple pages, vs. accessing a piece of information from one page. In case of a complex task requiring a multitude of pages to be accessed, the number page visits will positively predict task performance. In a simple task, however, where only one page needs to be accessed, a large number of page visits is not task-appropriate behavior and is likely to be negatively associated with performance (interaction i1 in Figure 1).

1.2.2 Predicting navigation behavior. Navigation behavior according to the model is predicted by the task (see path $\mathrm{p} 3$ in Figure 1). In general, students will adapt their navigation behavior according to task characteristics. For example, in a complex task that requires the student to integrate information from multiple pages, their navigation will entail more page visits and re-visits than in a simple task that needs retrieval of information that can be found on one single page. On the student level, navigation behavior is assumed to be predicted by online reading engagement. Different predictive patterns are expected for different dimensions of online reading engagement. As previous factor-analytic work has shown, online reading behaviors form to distinct dimensions, one that entails information-seeking activities (information engagement) and one that entails activities directed at social interaction and entertainment (social engagement, see Lee \& Wu, 2013; OECD, 2011, chapter 4). Both from theory and previous research, these two dimensions are assumed to have different associations with navigation behavior.

1.2.2.1 Information engagement and navigation behavior. Information seeking online reading activities (information engagement) comprises behaviors such as reading online news, using online encyclopedia or dictionaries, or using the internet to search for specific information. Activities such as these are frequently carried out while studying. Information seeking activities, such as searching the Internet for a specific topic for a school assignment, often require the student to carefully evaluate search results, to judge whether or not to follow the hyperlinks encountered, and to integrate the contents of the accessed documents into a coherent mental model. In line with these considerations, information 
engagement has been found to be positively associated with reading skill (Lee \& Wu, 2013), and online reading skill (OECD, 2011). There is however no evidence yet regarding the association between information engagement and navigation behavior. The model presented here assumes the following: Information engagement will strengthen a student's skill to navigate adaptively. A student high in information engagement will show a particular instance of navigation behavior, such as a high number of visits and re-visits to hypertext pages, according to task demands. Thus, in statistical terms, the association between information engagement and navigation behavior will be moderated by task demands (interaction i2 in Figure 1).

\subsubsection{Social engagement and navigation behavior. Social interaction oriented} online reading activities (social engagement), such as participating in social networks, Emailing, or playing collaborative games that involve reading online (e.g. Rama, Black, van Es, \& Warschauer, 2012; Thorne \& Black, 2007), have been reported to relate negatively to academic achievement and reading skill. Jacobsen and Forste (2011) found that use of social networking sites, use of cell phones for texting, and online gaming had all negative associations with GPA, and both Kirschner and Karpinski (2010), and Junco (2012a) found use of Facbook to be negatively related to students' GPA. Pfost, Dörfler and Artelt (2013) found use of E-Mail to be negatively associated with reading skill, even after controlling for a comprehensive set of background variables, including most importantly reading skills measured two years earlier. Lee and $\mathrm{Wu}(2013)$ found a negative association between social entertainment online activities and reading skill. Their measure of social entertainment online activities comprised use of Collaborative Online Games, E-Mail, Chat, Social Networking sites, and maintaining a personal Website or Blog.

What factors might contribute to these negative associations? Social interaction oriented online reading activities are often carried out in a playful manner, and compete with studying for time and cognitive resources. For instance, both Jacobsen and Forste (2011) and 
Junco (2012b) found the use of Facebook to be negatively associated with student engagement and time spent for class preparation. Social networking sites are often used in parallel to another task (Kirschner \& Karpinski, 2010). Calderwood, Ackerkman and Conklin (2014) observed 60 students' multitasking behavior during a 3-hour homework session. They found use of the computer and mobile phone for communication (e.g. reading e-mail) to be amongst the most prevalent multitasking activities. Multitasking is bound to impair comprehension and learning (e.g. Fox, Rosen and Crawford, 2008; Sana, Westen \& Cepeda, 2013). Especially in online reading, a careful selection of reading materials is crucial to comprehension and learning outcomes, which also means discarding non-relevant materials (e. g. Lawless \& Kulikowich, 1996; Salmerón et al., 2006). Speaking to this, Alloway and Alloway (2012) found that in a go/no-go task, Social media heavy users were more likely to be distracted by no-go stimuli, i.e. stimuli they were supposed not to attend to.

To date however, there is no evidence that links social engagement to navigation. From the considerations and studies cited above it would seem that students high in social engagement are less prepared for the cognitively demanding task of when to access pages while reading online, and which pages to discard. Thus, in contrast to what is expected for information engagement, students high in social engagement can be expected to act less adaptively than students low in social engagement (interaction i3 in Figure 1).

\subsection{The present research}

Neither the idea that navigation predicts digital reading performance conditional on task demands over and above reading skill, nor the idea that information and social engagement predict navigation differently have been addressed empirically (to the best of the author's knowledge). The present research aims at a contribution to filling in these gaps. This article presents two analyses, the first of which examines how online reading engagement relates to navigation behavior, and the second examines how navigation behavior relates to digital reading task performance. Other than in previous studies that utilized data from the 
Digital Reading Assessment (e.g. Lee \& Wu, 2013) individual task completion processes, and outcomes, serve as the primary unit of analysis, in accordance with the task layer in the model of online reading engagement (Figure 1). This makes it possible to actually predict performance from behavior, as for each individual task it is recorded how an individual student engages with this task before giving a correct or incorrect response. Clearly, however, prediction here does not imply causation.

As an indicator of navigation, the number of visits to task-relevant pages, including revisits, is considered. This indicator depicts information access behavior, which is one aspect of navigation, and can be assumed to predict performance conditional on task demands. Consider a task that needs visiting many pages (high access demands task). In such a task it is beneficial to frequently visit, and re-visit relevant pages. Frequent visits in this case will mean that a student comprehensively covers the materials, as is shown by a high correlation of this variable on the student level with the percentage of relevant pages accessed. Across countries and economies, this correlation ranged from .69 to $.91(M d=.83)$. Furthermore, if the student is engaged with a complex stimulus, re-visiting relevant pages can be a useful strategy. This is because in such tasks, information from multiple pages has to be integrated that will be difficult to bear in mind simultaneously. In line with this reasoning the number of visits to relevant pages has positive associations with learning outcomes in high access demands tasks (e.g. Naumann et al., 2008; Puntambekar et al., 2003).

Consider in contrast a task that needs visiting only few, or even only one single page (low access demands task). In such a task, frequent visits to relevant pages will result from behavior that is not directed at task completion by moving back and forth between relevant and non-relevant pages. Visiting and re-visiting task-relevant pages according to task demands is a strong determinant of learning outcomes (Gräsel, Fischer, \& Mandl, 2001; Naumann, Richter, Flender, Christmann, \& Groeben, 2007; Naumann et al., 2008; 
Niederhauser, Reynolds, Salmen, \& Skomolski, 2000; Puntambekar \& Stylianou, 2005; Richter, Naumann, \& Noller, 2003).

The questions (1) how access behavior relates to online reading engagement and task demands, and (2) how digital reading performance relates to access behavior, are addressed using data from the 2009 PISA Digital reading assessment. The present research thus uses the strength of large scale data bases that provide large samples and generalizability across countries and cultures. This is especially important, as different cultures might have a different take on interacting on line (e.g. OECD, 2011; Zhang, Ordóñez de Pablos, \& Xu, 2014). At the same time, in the Digital Reading Assessment log files of students' task completion processes were recorded. Through this, processes such as access behavior can be analyzed at the level of individual tasks. This approach was traditionally only employed in small-scale lab studies.

\subsection{Hypotheses}

1.4.1 Access behavior. From the above model of online reading engagement it follows that online readers adapt their navigation behavior to the task at hand. This means that there is an effect of access demands, i.e. the number of relevant pages within a task, on access behavior, i.e. the number of visits and re-visits to task-relevant pages (Hypothesis 1). The model also posits that especially readers who are regularly engaged in information seeking activities, such as reading online news or using online encyclopedia, adapt their behavior to task demands. We can thus assume a positive interaction of information engagement and access demands (Hypothesis 2a). According to this reasoning, in high access demands tasks, higher information engagement will be associated with more information access (Hypothesis 2b). In low access demands tasks, in contrast, higher information engagement should be associated with less information access (Hypothesis 2c).

A reverse pattern is expected for social engagement, i.e. the engagement in social interaction related online reading activities, such as using online forums or virtual 
communities. A negative interaction between access demands and social engagement is assumed (Hypothesis 3a): In high access demands tasks, higher social engagement according to this reasoning is associated with less information access (Hypothesis $3 b$ ). In low access demands tasks, in contrast, higher social engagement will be associated with more information access (hypothesis 3c).

1.4.2 Prediction of task performance. The model of online reading engagement assumes a positive association between print reading skill and the odds of succeeding in a digital reading task (Hypothesis 4). Over and above the effect of print reading skill, a positive effect of access behavior on performance in digital reading tasks is expected (Hypothesis 5). This effect however is assumed to be qualified by access demands. A positive interaction is expected between access behavior and access demands (Hypothesis 6a). This interaction is assumed to take on a positive sign since in high access demands tasks, a positive association of access behavior and performance is expected (Hypothesis 6b), while a negative association is expected in low access demands tasks (Hypothesis 6c).

\section{Method}

\subsection{Subjects}

Subjects were those students that participated in the PISA 2009 digital reading assessment for whom complete and valid log files were obtainable, who came from those countries and economies that also participated in the ICT familiarity questionnaire, and for whom information on information and social online reading engagement was available $(N=$ 29,395). Drop-out due to non-availability of log files was low, and amounted to $1.3 \%$ (or 417 cases) of the original sample in total, and $0 \%$ to $4.6 \%$. Detailed demographic information is provided in Online Supplement Table 1.

\subsection{Materials and Instruments}

2.2.1 Digital Reading Performance. Students' performance in each digital reading task was measured through their graded task responses in the PISA 2009 Digital Reading 
Assessment (0 indicating no credit, 1 indicating credit). The Digital Reading Assessment comprised of 29 items, distributed across nine units. A unit consisted of a text stimulus and between one and four tasks. Each student received six units, comprising either 18 or 19 tasks. The tasks presented online text materials in a simulated browser environment that mimicked typical features of commercial web browsers such as Internet Explorer, Firefox, Opera, or Chrome (see Figure 2). The texts represented genres typical for online text at the time the assessment was conceived (April-October 2007), such as blogs, websites, e-mails, or forums. Each task was situated in a hypertext environment consisting of multiple texts and pages, e.g. a search engine results page linking several web sites, which comprised multiple pages. Navigation was required to different degrees in different tasks. Digital reading tasks varied as to the number of task-relevant pages within a stimulus. A page was considered task-relevant (a) if it contained information that was required to complete a task, (b) if it contained information that could be assumed helpful to complete a task, or (c) if it was needed to achieve the navigation required for the task (see OECD, 2011, p. 91). The Unit "I want to help" for example features a girl named "Maika". Maika wants to take up a volunteer job and writes about this intention in her blog. In task 4 of this unit, students are asked to find, and recommend to Maika, a volunteering opportunity that fits her requirements, and explain to her in an E-mail why they think that this opportunity is suitable. To achieve this, they have to use a website from a non-profit organization called 'I want to help', that can be accessed through a link in Maika's blog entry. On the Home page of this website, a link has to be accessed leading to a page where four current opportunities are offered, two of them fitting, and two of them not fitting Maika's requirements. Each of the opportunities can be seen in further detail by clicking a link next to it. Each opportunities page has a link labeled 'E-mail this opportunity to a friend', that opens a form where an E-mail message can be entered and sent. In this task, the following pages are considered task-relevant: (1.) Maika's blog. This page contains information students have to use concerning Maika's requirements. (2.) The 'I 
want to help' website's Home page. This page is required for navigation. (3.) A page 'About' where Maika introduces herself as to her personality and interests. This page features information that is relevant to judge one of the volunteering opportunities as being suitable for Maika. It does not have to be used to solve the task, but is relevant for one out of two suitable task solutions. (4.) A page 'Latest opportunities' within the 'I want to help' site linking the various volunteering opportunities. This page is both required for navigation and contains information on which two of the four opportunities can already be discarded as not fitting with Maika's specifications. (5.) and (6.) The 'opportunity details' pages for each of the two suitable volunteering options. Either one of these two pages has relevant information, indicating that the respective opportunity fits with Maika's requirements. (7.) and (8.) The 'send e-mail pages' for each of the two fitting opportunities. (9.) and (10.) A confirmation page, where, for each opportunity, students can either eventually send their message, or choose to further edit it using an "Edit your message" button. (11.) A page confirming the Email has been sent. (12.) and (13.) A "Site map", and a "Resources" page within the "I want to help" website. These pages are not required for task completion. They can, however, from their labels, be considered useful for navigation ("Site map") or considered offering further opportunities ("Resources"). Thus, this task comprises 13 relevant pages. The shortest possible navigation route requires visiting seven pages. It is important to note that in each task the text stimulus offered many pages that were not relevant, but accessible. In the sample task, these were e. g. a page where a student could directly contact the 'I want to help' organization for taking up a volunteering job for themselves (rather than recommending it to someone else). This page was not relevant as students were explicitly instructed to recommend a volunteering opportunity to Maika. Also, the pages offering the two not suitable options were not relevant. This was because students could identify these two pages offered non-suitable options without actually visiting them. This task, as well as other released tasks from the 
Digital Reading Assessment can be seen and interactively operated at http://erasq.acer.edu.au/ $(9 / 2 / 2013)$.

2.2.2 Access demands. Access demands were measured through counting the number of task-relevant pages in each task.

2.2.3 Access behavior. Access behavior was measured through the number of visits to task-relevant pages taken by a student, including re-visits. This variable was obtained through analyzing $\log$ files of student behavior.

\subsubsection{Online information reading engagement (information engagement). This}

variable was measured through four items from the PISA 2009 Student Questionnaire asking students how regularly they performed online reading activities targeted at searching for specific information. These items were 'Using a dictionary', 'Reading News', 'Search the Internet for a particular topic', and 'Search the Internet for practical information'. In accordance with procedures used in PISA, items were scaled according to the partial credit model, providing a Weighted Least Squares estimate (WLE) for each student's online information reading engagement (see OECD, 2012, p. 284 ff for details). ACER Conquest (Wu, Adams, \& Haldane, 2007) was used for scaling. The WLE reliability was .73, Cronbach's $\alpha$ was .75.

2.2.4 Online social interaction reading engagement (social engagement). This variable was measured through five items from the PISA 2009 ICT questionnaire, asking students how regularly they performed online reading activities tied to social interaction. These items were 'Use e-mail', 'Chat online', 'Publish and maintain a personal website, weblog or blog', 'Play collaborative online games', and 'Participate in online forums, virtual communities or spaces'. These items were scaled according to the partial credit model. The WLE reliability for these five items was .56, Cronbach's $\alpha$ was .68.

2.2.5 Reading skill. This variable was measured through the PISA 2009 print reading assessment. This assessment comprised a total of 131 print reading items, distributed across 
37 units. These units were distributed across 13 booklets, which also contained items from the PISA science and mathematics assessments. The PISA reading assessment covers reading skill in different text formats (continuous and non-continuous), situations (personal, public, occupational and educational contexts), and aspects or cognitive operations (accessing and retrieving, integrating and interpreting, and reflecting and evaluating textual information, see OECD, 2009, for details). Reading skill was scaled according to the Rasch model. The WLE reliability was .80 (OECD, 2012, p. 194).

2.2.6 ICT availability at home. This variable was measured through eight items from the ICT familiarity questionnaire that asked for a number of ICT devices whether these were available in students' homes, and whether the students used them. These devices were a 'desktop Computer', 'a portable laptop', an 'Internet connection', a 'video games console', a 'cell phone', an 'mp3/Mp4 player', a 'printer', and a 'USB (memory) stick'. WLEs provided in the PISA 2009 data base were used. For the present sample, Cronbach's $\alpha$ was .64.

2.2.7 ICT availability at school. This variable was measured through five items from the ICT familiarity questionnaire that asked for a number of ICT devices whether these were available to students at school, and if so, whether they used them. These devices were a 'desktop computer', a 'portable laptop', an 'Internet connection', a 'printer', and a 'USB (memory) stick'. WLEs were used that are provided in the PISA 2009 data base. For the present sample, Cronbach's $\alpha$ was .66.

2.2.8. Social-economic status (SES). SES was measured using the HISEI index. This index gives the highest occupational status among the two parents of a student (see Ganzeboom, De Graaf \& Treiman, 1992, for details).

\subsection{Procedure}

Students were tested in schools during school hours. First, they completed the paperpencil assessment that assessed students' reading skill, as well as their mathematics and science skills, which lasted for two hours. Afterwards, they completed the Student 
questionnaire, which collects information on students' background (e.g. gender, socioeconomic background), and reading attitudes, practices, and strategies. After this, they completed the ICT familiarity questionnaire, asking for students' availability and use of ICT, both at home and at school, as well as their attitudes towards, and confidence in using ICT. Then the computer-based Digital Reading Assessment was administered. For this assessment, a minimum of 10 students per school were sampled from those students who had also completed the printed assessment. The Digital Reading Assessment took 40 minutes. Before the actual test, students received a tutorial where they learned how to operate the interface and simulated web browser. The assessment was carried out in a secure test environment. Data were collected using USB devices. Throughout the assessment, students' responses and the sequence of actions they took through test completion (clicks) were recorded in log files.

\subsection{Data analysis and modeling approach}

Analyses were carried out country-wise. This provided 17 independent estimates for each effect.

2.4.1 Predicting access behavior. To predict access behavior, a linear mixed model (LMM) approach was used. In an LMM, in addition to the fixed effects of the predictors, random effects are included. In the present case, a random person effect (corresponding to person-specific access behavior), and a random item effect (corresponding to item-specific access behavior) were included. To model a random person effect in the present analyses is critical for the following reason. A model without the specification of such an effect would assume that all the between-persons variance can be completely explained by person level covariates (online reading engagement and control variables). Put differently, it would be assumed that after controlling for person level covariates, observations coming from the same person were independent from one another, which is an unrealistic assumption.

The same logic applies to items. A model not specifying a random effect for items would make the assumption that all the between-items variance can be explained by item 
level covariates (here: access demands). In other words, such a model would assume that after controlling for item level covariates, observations coming from the same item are independent from one another. This, again, is an unrealistic assumption. A random effect for schools was included in addition to account for students being nested within schools.

\subsubsection{Predicting digital reading performance. To predict digital reading}

performance a Generalized Linear Mixed Model approach (GLMM, see Baayen, Davison, \& Bates, 2008) was used. A GLMM differs from an LMM in that ordered categorical outcomes are modeled. To achieve this, the ordered categorical outcome that is assumed to have some specified distribution is tied to the linear combination of predictors through some link function. In the present case the log-transformed odds ratio (log odds) of a correct response was regressed on the set of hypotheses-relevant predictors and control variables, assuming a binomial distribution of the response variable. Effects of predictor and control variables were entered as fixed effects. In addition, random effects for persons, items, and schools were specified for the same reasons as in the LMM for access behavior.

\subsubsection{Aggregating results across countries: Random-effects meta-analysis}

Researchers might be interested in how effects turn out averaged across countries, as well as in between-country variation. To estimate both an average effect, and the variance of effect sizes between countries, random effects meta-analysis was employed (see Hedges \& Vevea, 1998). Random-effects meta-analysis decomposes an effect into an overall population effect, which is independent of the specific studies (here: countries), a study specific effect (here: country specific effect), that occurs due to the particulars of the respective studies (e. g. methods, materials, participants), and an effect of sampling variance. It thus separates the variance in effect sizes due to study-specific effects $\tau^{2}$, from sampling variance $\sigma^{2}$. Through this, inferences can be made that go beyond the studies in the analysis, and the variance of the study-specific effects $\tau^{2}$ can be tested for significance. Random-effects meta-analysis lends itself to the present research for two reasons. First, the goal of the present research is to 
generalize across countries beyond the ones that participated in the PISA 2009 Digital Reading Assessment. Second, it is of interest to see whether the obtained effect vary across countries and economies to a degree that cannot be explained by sampling variance alone. As a measure of an effects' variability, the ratio of the variance of study-specific effects to the total variability $I^{2}$ is reported, i.e. the ratio of $\tau^{2} /\left(\tau^{2}+\sigma^{2}\right)$. To provide a measure of an effect's variability in absolute terms, the $95 \%$ confidence interval is reported for each effect size in addition.

\section{Results}

\subsection{Predicting access behavior}

To predict access behavior, a linear mixed model with random effects for students, items, and schools was specified. The following LMM was estimated:

$$
\begin{aligned}
& \text { access behavior }=\beta_{0}+\beta_{1}(\text { information engagement })+\beta_{2}(\text { social engagement }) \\
& +\beta_{3}(\text { access demands })+\beta_{4}(\text { information engagement } \times \text { access demands }) \\
& +\beta_{5}(\text { social engagement } \times \text { access demands })+\beta_{6}(\text { reading skill }) \\
& +\beta_{7}(\text { ICT at home })+\beta_{8}(\text { ICT at school })+\beta_{9}(\text { gender })+\beta_{10}(\mathrm{SES}) \\
& +b_{0 \mathrm{i}}+\mathrm{b}_{0 \mathrm{p}}+\mathrm{b}_{0 \mathrm{~s}}
\end{aligned}
$$

In this model, $\beta_{0}$ is the intercept. $\beta_{1}$ to $\beta_{5}$ are fixed effects of the hypothesis-relevant predictors and their interactions. $\beta_{6}$ to $\beta_{10}$ are fixed effects of the control variables. $b_{0 \mathrm{i}}$ is the random item-specific adjustment to the intercept (relevant page visits in items i). $b_{0 p}$ is the random person-specific adjustment to the intercept (relevant page visits by person $p$ ). $b_{0 s}$ is the random school-specific adjustment to the intercept (relevant page visits in school s). Gender was entered as a dummy coded variable with boys as the reference group. All other predictor and control variables were standardized country-wise. The dependent variable access behavior was left in its original metric (number of relevant page visits). Regression coefficients can thus be interpreted as expected change in the number of relevant page visits 
per one standard deviation change in the respective predictor variable. All predictors were entered simultaneously. Models were estimated using the $\mathrm{R}$ environment ( $\mathrm{R}$ development Core Team, 2012) with the package lme4 and the function lmer (Bates, Maechler, \& Bolker, 2012). Meta-analyses were conducted using the package metafor (Viechtbauer, 2010).

As a measure of effect size, the model-expected difference in the number of relevant page visits between a student or a task low (-1 SD) vs. a student or a task high $(+1 S D)$ in each predictor variable is reported (e.g. Schielzeth, 2010). These effect sizes can be interpreted as follows. OECD (2011) found that aggregated across all tasks completed by a student, each additional visit to task-relevant pages per student accounted for an increase of 2.4 points on the PISA reading scale. With 18 or 19 tasks in the assessment per student, 1 additional visit to task relevant pages per task would amount to an increase in the predicted score of between 43 and 45 on the PISA scale, corresponding to a large effect. Thus, as a rule of thumb, effect sizes of 1 or above can be described as large, effect sizes between 0.5 and 1 can be described as medium, and effect sizes smaller than 0.5 can be described as small.

An alpha-level of .05 was chosen. When hypotheses implied an effects' direction, tests were one-tailed. Because each country-specific model tested five hypotheses on the same data set each, Bonferroni correction was applied, arriving at an operational alpha level of .01. In accordance with suggestions by Card (2012), no Bonferroni correction was applied for metaanalytic tests.

3.1.1 Random effects. In each country, access behavior varied mostly between tasks, next between students, and least between schools. Estimates of random effects for tasks, students and schools are provided in Online Supplement Table 2.

3.1.2 Fixed effects. Only results for the hypothesis-relevant fixed effects are reported. Estimates of the effects for the control variables are provided in Online Supplement Table 3.

3.1.2.1 Access demands. In each country, there was a significant effect of access demands (see Table 2 column 3). These effects were large (Table 2, column 4). In general, 
students adapted their access behavior to the task: The higher a task was in access demands, i.e. the more visits to task relevant pages it required, they more frequently students visited task-relevant pages. Across countries and economies, the meta-analytically derived effect was $\beta=3.16(S E=0.13), \mathrm{z}=23.82, p<.05,95 \%-\mathrm{CI}=[2.90 ; 3.42]$. Thus, students made on average 3.16 more visits to task-relevant pages per one standard deviation increase in access demands. This result gives support to hypothesis 1 . The variance component was significant, $I$ $2=0.41, Q(16)=30.14, p<.05$.

3.1.2.2 Information engagement. For information engagement positive main effects were obtained, which turned out significant in 13 out of 17 countries (Table 2, column 5). When there were effects of information engagement, these were small (Table 2, column 6). Across countries, the meta-analytically estimated effect was $\beta=0.08(S E=0.01), z=9.75, p$ $<.05,95 \%-\mathrm{CI}=[0.06 ; 0.10]$. The variance component was insignificant, $I^{2}=0.29, Q(16)=$ $23.65, p>.05$. This indicated that information engagement had a positive, albeit very small, association with access behavior that showed no variation across countries over and above sampling variance. Thus, students who routinely performed information-related online reading tasks, such as using an online-encyclopedia, tended to perform more visits and revisits to task-relevant pages.

In each country, with only one exception, the effect for information engagement was qualified by a significant interaction with access demands (Table 2, column 7). This interaction was positive for each country, meaning that the association of access demands with access behavior was more positive in students who reported a higher degree of information engagement. These interactions were of small to medium size (Table 2, column 8). Thus, as expected, the degree to which students' information-related online reading behavior was associated with their visiting task-relevant pages was conditional on task demands. Meta-analytically, the interaction between access demands and information engagement was $\beta=0.17(S E=0.02), \mathrm{z}=9.35, p<.05,95 \%-\mathrm{CI}=[0.13 ; 0.20]$. This result 
gives support to hypothesis $2 \mathrm{a}$. The variance component was significant, $I^{2}=0.93, Q(16)=$ $264.58, p<.05$. This indicated that, while the interaction was positive in general, it differed across countries and economies.

Simple slopes analyses revealed that in each country, there was a significant positive association of information engagement with information access in high access demands tasks, (see Table 3, column 2, and the left-hand white boxplot in Figure 3a). These effects were of medium to large size in ten, and of small to medium size in seven countries (Table 3, column 3). Across countries, this slope was significant, $\beta=0.25(S E=0.03), z=10.01, p<.05,95 \%$ $\mathrm{CI}=[0.20 ; 0.29]$, and amounted to a medium-sized effect (see the upper dashed line in Figure 3b). Thus, in tasks that required the frequent visit and re-visit of task-relevant pages, students who regularly engaged with information-related online texts (information engagement) acted more in line with the tasks' information access demands. This result gives support to hypothesis $2 \mathrm{~b}$. The associated variance component was significant, $I^{2}=0.88, Q(16)=148.94$, $p<.05$. This indicated that while the association of information engagement with access behavior in high access demands tasks was positive in general, the steepness of the slope varied across countries.

In contrast, for low access demands tasks, information engagement was negatively associated with access behavior (see Table 3, column 4 and the right-hand white boxplot in Figure 3a). This negative association was significant for 13 countries. These effects were small (see Table 3, column 5). The meta-analytically derived association was $\beta=-0.09$ (SE $=$ $0.01), z=-6.39, p<.05,95 \%-\mathrm{CI}=[-0.12 ;-0.06]$, amounting to a small effect (see the lower dashed line in Figure 3b). Thus, in tasks that did not require the frequent visit and re-visit of task-relevant pages, students who regularly engaged with information-related online texts acted more parsimoniously and thus again in line with the tasks' information access demands. This result gives support to hypothesis $2 \mathrm{c}$. The associated variance component was significant, $I^{2}=0.63, Q(16)=47.40, p<.05$. Thus, the association of information 
engagement with access behavior in low access demands tasks differed across countries. All in all, this means that students who regularly engage with information-related online texts (information engagement) showed task-adaptive navigation: They aligned their visiting and re-visiting task relevant pages to each task's requirements. Students who were less strongly engaged with information-related online media in contrast showed less adaptive navigation.

3.1.2.3 Social engagement. Other than information engagement, social engagement had weak associations with access behavior (Table 2, columns 9-10). Meta-analytically, the effect was insignificant, $\beta=0.01(S E=0.01), z=0.68, p=.50,95 \%-C I=[-0.01 ; 0.20]$. The variance component was insignificant, $I^{2}=0.14, Q(16)=17.08, p>.05$. Thus, the frequency with which students engaged with social-related online texts such as social networks (social engagement) was not associated with their visiting and re-visiting task-relevant pages.

In nine countries there was a negative interaction of social engagement with access demands (Table 2, column 11). Thus, in these countries, students who regularly engaged with social-related online texts were less prone to align their visiting and re-visiting of taskrelevant pages to each task's information access demands. These effects were however small (Table 2, column 12). The meta-analytically derived effect size was negative, $\beta=-0.03,(S E=$ $0.02), \mathrm{z}=-1.80, p<.05,95 \%-\mathrm{CI}=[-0.06 ; 0.00]$, and associated with a significant variance component, $I^{2}=0.92, Q(16)=184.50, p<.05$. These results give some support to hypothesis 3a, although the effect was very small, and was not found in each country.

Simple slopes analyses found a negative effect of social engagement in high access demands tasks in six countries (Table 3, column 6, and the left-hand grey box in Figure 3a). These effects were small (Table 3, column 7). In these countries, students high in social engagement made fever visits and re-visits to task-relevant pages in high access demands tasks. Across countries, this slope was not significant, $\beta=-0.02(S E=0.02), z=-1.05, p>$ $.05,95 \%-\mathrm{CI}=[-0.06 ; 0.02]$ (see the upper straight line in Figure $3 b)$. There was variation 
between countries, $I^{2}=0.85, Q(16)=101.39, p<.05$. This result contradicts hypothesis $3 \mathrm{~b}$, which had predicted a negative effect of social engagement in high access demands tasks.

For low access demands tasks, social engagement had a positive effect in six countries (see Table 3, column 8, and the right-hand grey box in Figure 3a). These effects were small (Table 3, column 9). Meta-analytically, positive effect was found, $\beta=0.04(S E=0.01), z=$ $3.27, p<.05,95 \%-\mathrm{CI}=[0.02 ; 0.06]$, which was small (see the lower straight line in Figure $3 b)$. This effect was associated with significant between-county variability, $I^{2}=0.52, Q(16)=$ $35.36, p<.05$. This result gives some support to hypothesis $3 \mathrm{c}$.

Taken together these results suggest the following: In line with hypothesis 1, students generally adapted their information access behavior to the task. The more visits to task relevant pages a task required, the more such visits and re-visits were performed by students. These effects were large. This adaptation was more pronounced for students high in information engagement as opposed to students low in information engagement. Thus, students who regularly engaged with information-related online texts such as news, or onlineencyclopedia, performed more visits and re-visits to task relevant pages than their peers who were less engaged with information related online reading, but only if this was required by the task. This was indicated by the interaction between access demands posed by the task, and information engagement, as suggested by hypothesis $2 \mathrm{a}$. This interaction meant that a positive association between information engagement and access behavior was confined to high access demands tasks tasks, as suggested by hypothesis $2 \mathrm{~b}$. In low access demands tasks, the relation was negative, as suggested by hypothesis $2 \mathrm{c}$.

Such an association with task-adaptive navigation behavior was not found for social engagement. On the contrary, in nine individual countries and economies as well as overall, students who regularly engaged with social-related online media, such as social networks, were less adaptive in their navigation, as suggested by hypothesis $3 \mathrm{a}$. This interaction meant that in low access demands tasks, students high in social engagement more frequently visited 
task-relevant pages. It did not mean that students high in social engagement less frequently visited task-relevant pages in high access demands tasks, disconfirming hypothesis $3 b$.

\subsection{Predicting task performance}

The analyses provided in the last section showed that information engagement predicts adaptive content access behavior in 15 year olds' use of digital text, while social engagement does not. The ultimate goal in digital reading however is not content access per se, but performance on a task that comprises content access, as well as text and graph comprehension. Thus the question stands how task-adaptive content access behavior predicts task performance in digital reading, in addition to a student's skill in print reading. To answer this question, task performance was regressed on print reading skill, access behavior, access demands, and most importantly, the interaction of access behavior and access demands. If it were adaptive access behavior that predicted performance in digital reading tasks, not only content access per se should be related to digital reading performance, but especially content access that fits task demands. Gender, socio-economic status, and ICT availability at home and at school were controlled for. In addition, information engagement, social engagement, and their interaction with access demands were controlled for. The following GLMM was estimated:

$$
\begin{aligned}
& \ln [p /(1-p)]=\beta_{0}+\beta_{1}(\text { print reading skill })+\beta_{2}(\text { access demands }) \\
& +\beta_{3}(\text { access behavior })+\beta_{4}(\text { access behavior } \times \text { access demands }) \\
& +\beta_{5}(\text { information engagement })+\beta_{6}(\text { social engagement }) \\
& +\beta_{7}(\text { information engagement } \times \text { access demands }) \\
& +\beta_{8}(\text { social Engagement } \times \text { access demands }) \\
& +\beta_{9}(\text { ICT at home })+\beta_{10}(\text { ICT at school })+\beta_{11}(\text { gender })+\beta_{12}(\mathrm{SES}) \\
& +\mathrm{b}_{0 \mathrm{i}}+\mathrm{b}_{0 \mathrm{p}}+\mathrm{b}_{0 \mathrm{~s}}
\end{aligned}
$$

In this model, $\mathrm{p}$ is the probability of successful performance on a digital reading task. $\beta_{0}$ is the intercept. $\beta_{1}$ to $\beta_{4}$ are fixed effects of the hypothesis-relevant predictors, and their 
interactions. $\beta_{5}$ to $\beta_{12}$ are fixed effects of the control variables. $b_{0 \mathrm{i}}$ is the item-specific adjustment to the intercept, which is modeled as a random variable (i.e., the easiness of item i). $b_{0 p}$ is the person-specific adjustment to the intercept, which is modeled as a random variable (i.e., the digital reading skill of person $p$ ). $b_{0 s}$ is the school-specific adjustment to the intercept, which is modeled as a random variable (i.e. digital reading performance in school s). Gender was entered as a dummy coded variable with boys as the reference group. All other predictor and control variables were standardized country-wise. Once again, analyses were carried out per country, and country-specific results were integrated meta-analytically.

As a measure of effect size, the model-expected difference in the probability of scoring correctly on a task between a student or a task low (-1 SD) vs. a student or a task high $(+1 S D)$ in each predictor variable is reported. These differences in probabilities can be interpreted referring to PISA reading proficiency levels (see OECD, 2012). In PISA, tasks are scaled according to the Rasch-Model, which puts student skills and task difficulties on a common scale. A student scoring at the bottom of a given PISA reading proficiency level will have a $50 \%$ chance to solve a task with a corresponding difficulty. A student at the top of each proficiency level will have a chance of $70 \%$ to solve a task with a difficulty corresponding to the bottom of the respective proficiency level. Thus, it takes a student to move up a whole level of reading proficiency to increase their probability of solving a given reading task by .20. Following this, a .20 difference in model-predicted probabilities can be interpreted as a large effect. A .10 difference can be interpreted as a medium-sized effect, and a .05 difference can be interpreted as a small effect.

An alpha-level of .05 was chosen. Since all hypotheses implied effects' directions, tests were one-tailed. Because each country-specific model tested four hypotheses on the same data set each, Bonferroni correction was applied, arriving at an operational alpha of .0125. Once more, no Bonferroni correction was applied for the meta-analytic tests of effects (Card, 2012). 
3.2.1 Random effects. In each country, digital reading performance varied mostly between tasks, next between students, and least between schools. Estimates of random effects for tasks, students and schools are provided in Online Supplement Table 2 (columns 4-7).

3.2.2 Fixed effects. Only results for hypothesis-relevant fixed effects are reported. Estimates of the control variables' effects are provided in Online Supplement Table 4.

3.2.2.1 Print reading skill. As expected, print reading skill was positively associated with digital reading performance (Table 4, column 3). These effects were large (Table 4, column 3). The difference in the probability of solving a digital reading task between students low (-1 SD) or high (+1 SD) in print reading skill was .20 or larger in all but two countries. Meta-analytically a significant effect was obtained, $\beta=0.71(S E=0.03), \mathrm{z}=26.73, p<.05$, $95 \%-\mathrm{CI}=[0.66 ; 0.77]$. The associated variance component was significant, $I^{2}=0.95, Q(16)=$ $304.36, p<.05$.

3.2.2.2 Access demands. Access demands had no significant association with task performance in any individual country (Table 4, columns 5 and 6). Effects were, however, consistently negative, and significant meta-analytically, $\beta=-0.18(S E=0.05), z=-3.57, p<$ $.05,95 \%-C I=[-0.27 ;-0.08]$. There was no significant between-country-variation, $I^{2}=0.01$, $Q(16)=3.58, p>.05$. The meta-analytically derived effect size corresponded to a modelpredicted difference of -.07 in the probability of correctly solving a task with high vs. low navigation demands, amounting to a small to medium sized effect.

3.2.2.3 Access behavior. Access behavior was positively predictive of task performance in each country (Table 4, column 7). These effects varied from medium to large (Table 4, column 8). Meta-analytically, the effect was $\beta=0.35(S E=0.02), \mathrm{z}=16.62, p<$ $.05,95 \%-\mathrm{CI}=[0.31 ; 0.39]$. Effects of access behavior varied significantly between countries, $I^{2}=0.76, Q(16)=73.84, p<.05$. Thus, students who performed more visits and re-visits to task-relevant pages performed better in digital reading, after controlling for both reading skill and the control variables in the model. This result gives support to hypothesis 5 . 
Most importantly however, the effect of access behavior was qualified by a significant interaction of access behavior with access demands in each county (Table 4, column 9). These interactions were of medium to large size (Table 4, column 10). The meta-analytically derived effect size for this interaction was $\beta=0.16(S E=0.01), \mathrm{z}=13.84, p<.001$. The associated variance component was significant, $I^{2}=0.66, Q(16)=50.77, p<.05$. Thus, the effect of students visiting and re-visiting task-relevant pages was conditional on the degree to which tasks required this kind of navigation behavior. This result gives support to hypothesis $6 \mathrm{a}$. The positive sign of the interaction meant that access behavior was a stronger predictor of task performance in high access demands tasks than in low access demands tasks.

This was further corroborated by simple slopes analyses. In high access demands tasks, there was a positive association of access behavior and performance in each country (see Table 5, column 2, and the left-hand boxplot in Figure 3c). These effects were associated with model-predicted differences in the probabilities of correctly solving a task of above .20 for nine countries, and above .15 for another seven countries, indicating a large effect (see Table 5, column 3). Meta-analytically a large effect of $\beta=0.51(S E=0.02), z=29.74, p<$ $.05,95 \%-C I=[0.47 ; 0.54]$ was derived (see the straight line in Figure $3 \mathrm{~d}$ ), which was associated with significant between-country variance, $I^{2}=0.82, Q(16)=95.23, p<.05$. This result gives support to hypothesis $6 \mathrm{~b}$.

In low access demands tasks, the association between access behavior and performance was considerably weakened, but came out positive in each country (see Table 5, column 4, and the right-hand boxplot in Figure 3c). Meta-analytically, access behavior was still positively associated with task performance in low access demands tasks, $\beta=0.19(S E=$ $0.03), \mathrm{z}=6.34, p<.05,95 \%-\mathrm{CI}=[0.13 ; .24]$ (see the dashed in line in Figure $3 \mathrm{~d})$, and varied significantly between countries, $I^{2}=0.71, Q(16)=60.94, p<.05$. This result contradicts hypothesis $6 \mathrm{c}$, which predicted a negative association of access behavior with performance. 
All together these results suggest the following: Even after controlling for a comprehensive set of relevant background variables, and taking into account print reading skill, students' performance in digital reading tasks was predicted by the number of visits and re-visits to task-relevant pages. This relation was strong especially for tasks where content access behavior actually behavior meets task demands, i.e. in tasks that require the student to visit, and possibly re-visit, task-relevant pages to a large degree. In contrast, in tasks that did not require students to visit and re-visit task-relevant pages to a large degree, the number of visits to task-relevant pages was much less predictive overall. It should be noted that in low access demands tasks the relation between access behavior and performance still came out positive.

\section{Discussion}

The present results are the first to give evidence of an association between information seeking online reading engagement and adaptive navigation, specifically content access behavior, in digital reading. The opposite association was found for social interaction oriented online reading engagement: Students high in social interaction online reading engagement tended to behave less adaptively in digital reading. The present research also is the first to show how the association of content access behavior in digital reading is conditional on task demands. Although the predicted negative association between content access and task performance in low access demands tasks was not found, the interaction between task demands and access behavior was strong, and reliably found in 17 countries and economies, and it was in the expected direction: Digital reading performance was especially strongly predicted by access behavior in high access demands tasks, and much less so in low access demands tasks.

\subsection{Model of online reading engagement}

The present results thus confirm two important predictions made by the model of online reading engagement depicted in Figure 1. First, task-appropriate content access 
behavior in online reading predicted task performance, even after controlling for a comprehensive set of background variables, with reading skill among them. Thus, content access behavior is apparently one crucial aspect of digital reading that cannot be mapped upon a students' skill in reading generally. This result is in line with previous analyses that found navigation behavior to predict hypertext comprehension over and above reading skill (Naumann et al., 2008; OECD, 2011; Salmerón \& García, 2011).

Second, task-appropriate content access behavior was positively predicted by information engagement, while it is negatively predicted by social engagement. One possible explanation for this set of results is the mindsets, or motivational states, that govern the typical usage of social online media. While social networking sites or even interactive online games (e.g. Rama et al., 2012) might be used for educational purposes, mostly they are not (e.g. Junco, 2012b; Kirschner \& Karpinski, 2010). In other words, by using this media, students do not regularly cognitively engage in demanding tasks involving the thorough evaluation of hyperlinks as to their potential relevance for the task at hand. Thus, using social online media most likely does not result in developing the skills needed for task-adaptive navigation, or more specifically, content access behavior. In contrast, these skills are most likely practiced through the use of information oriented online media such as encyclopedia or news websites.

\subsection{Is information engagement beneficial to digital reading performance?}

Yes, it might be. The present research found evidence that information oriented online reading engagement positively relates to adaptive navigation. Specifically, students high in information engagement made more visits to task-relevant pages in high access demands tasks. Since visiting task-relevant pages is strongly predictive of task performance in high access demands tasks, it is likely that these students show improved digital reading performance as well. As OECD (2011, ch. 4) put it: "Most of the digital reading tasks [that were analyzed in this research, AUTHOR] call for searching-information strategies and 
navigation, skills that can be developed or reinforced by repeated contact with online searching-information practices" (p. 137). What is more, students high in information engagement behaved more parsimoniously when they encountered a low access demands task. In such a task, that requires visiting only one or two pages, students high in information engagement visited those one or two pages. What they apparently did not was to visit yet other pages that were not relevant to the task, and then navigated back, thereby unnecessarily increasing the count of visits to task-relevant pages.

\subsection{Is social engagement detrimental to digital reading performance?}

Probably not. What the present research showed was that high social online reading engagement was associated with less adaptive a style of navigation. However, metaanalytically, this did not mean that students high in social engagement fell short in high access demands tasks in terms of access behavior. Rather, in low access demands tasks, they did more than was necessary. Doing more than what is necessary in low access demands tasks however seems not harmful: In low access demands tasks, the association between the number of visits to task-relevant pages and performance was still positive. Thus it seems that while the less adaptive navigation done by students high in social engagement might impair the efficiency of their navigation, it does not harm the effectiveness. So on the one hand there may be concern that extensive use of e.g. social networking sites negatively impacts “traditional" academic achievement measures (e.g. Junco, 2012a; Pfost et al., 2013). This on the other hand does not mean that it also negatively impacts students' new literacy skills. Seemingly however, it is also not hugely beneficial.

\subsection{Educational implications}

As will be pointed out in more detail in section 4.5, the nature of the present data is correlational. This means that conclusions as to educational implications that rely on a causal interpretation of the effects reported in this article are tentative at best. This said, there appears to be some evidence that students might fail in digital reading that require accessing 
relevant pages to a certain degree specifically because they are unable to do the navigation that is required. This is indicated by the strong effect of the number of visits to relevant pages on task performance in high access demands tasks. This could mean that navigation and on line search skills, which many students are not in command of (e.g. Keil \& Kominski, 2013; OECD, 2011; Salmerón, Cerdán, \& Naumann, 2015) should receive more attention in formal education. This notion is also supported by the finding that students' reading engagement targeted at online information search has a positive association with task-adaptive information access behavior, which presumably comes from practice (see above, section 4.4). If this interpretation was correct, a process that now relies to a large degree on students' habitual on line information searching on line reading behaviors, the skills obtained from these habitual behaviors could be substantially strengthened if they were taught in classrooms.

\subsection{Limitations and final conclusions}

Most of the research that utilizes large scale data to answer substantive research questions is correlational. While using large scale data provides obvious advantages (large samples, cross-country comparability), cross-sectional designs pose obvious limitations to the conclusions that can be drawn from these data sets. While we can statistically predict students' adaptive navigation from their past online reading engagement, we do not know if in fact information seeking online reading engagement is causally relevant for adaptive navigation, as supposed by the model discussed in section 1.2. Maybe the latent ability to adaptively navigate is responsible both for a students' observable adaptive navigation and their observable online reading engagement. Most likely engagement and adaptive navigation have a reciprocal relation (see e.g. Stanovich, 1986; 2000). Also, large-scale assessment studies usually target well-defined populations, 15-year olds in the case of PISA. This of course means that although the samples provide good variation in terms of social background or intellectual ability, future research is needed to generalize the present findings to other age groups. 
A second limitation is that the present research did not dig deep into between-country variation. While some of the effects found in this study were consistent across countries, others were not. For example, while the interaction between social engagement and access demands meant that in nine countries highly socially engaged students navigated less adaptively, it meant the opposite for another four countries. Future studies should address this between-country variation in more detail, also as the number of countries that participate in the Digital Reading Assessment grows, thus providing a stronger data base for analyzing between-country variation (OECD, 2014).

A third and final limitation comes from the somewhat limited measure of both information seeking and social oriented online reading engagement. As pointed out in the writings of Guthrie and colleagues (e.g. Guthrie et al., 2012), reading engagement, and thus on line reading engagement, encompasses more than more or less frequently using certain types of online media. For example, in the present research no information was available as to for which purposes online texts such as news, or social networks, were used. In fact, while overall the use of social networking sites such as Facebook has negative associations with achievement and skill, the use of Facebook for a few very specific purposes, such as sharing interesting links, has indeed positive associations with academic achievement (Junco, 2012a). It will be worth wile to investigate in future studies the associations of using social online media for specific purposes with adaptive navigation and proficiency in digital reading. These analyses also might point at how social on line media can be utilized for teaching both traditional and new literacy skills. 


\section{References}

Afflerbach, P., \& Cho, B.-Y. (2008). Identifying and describing constructively responsive comprehension strategies in new and traditional forms of reading. In S.E. Israel, \& G.G. Duffy (Eds.), Handbook of research on reading comprehension (pp. 69-90). New York: Routledge.

Alloway, T. P. \& Alloway, R. G. (2012). The impact of engagement with social networking sites (SNSs) on cognitive skills. Computers in Human Behavior, 28, 1748-1754. DOI: $10.1016 /$ j.chb.2012.04.015

Amadieu, F., Tricot, A., \& Mariné, C. (2009). Prior knowledge in learning from a nonlinear electronic document: Disorientation and coherence of the reading sequences. Computers in Human Behavior, 25, 381-388. doi:10.1016/j.chb.2008.12.017

Baayen, R.H., Davidson, D.J. and Bates, D.M. (2008) Mixed-effects modeling with crossed random effects for subjects and items. Journal of Memory and Language 59, 390-412. DOI: $10.1016 /$ j.jml.2007.12.005

Bates, D., Maechler, M., \& Bolker, B. (2012). lme4: Linear mixed-effects models using S4 classes. R package version 0.999999-0. Retrieved January 14, 2014 from http://CRAN.Rproject.org/package $=1 m e 4$

Calderwood, C., Ackerman, P. L., \& Conklin, E. (2014). What else do college students 'do' while studying? An investigation of multitasking. Computers \& Education, 7519-29.

DOI:10.1016/j.compedu.2014.02.004

Card, N. A. (2012). Applied meta-analysis for social science research. New York: Guilford Press.

Cress, U., \& Knabel, O. B. (2003). Previews in hypertext: Effects on navigation and knowledge acquisition. Journal of Computer Assisted Learning, 19, 517-527. DOI: 10.1046/j.0266-4909.2003.00054.x 
Fox, A. B., Rosen, J. \& Crawford, M. (2008). Distractions, distractions: Does instant messaging affect college students' performance on a concurring reading comprehension task? Cyberpsychology and Behavior, 12, 51-53. DOI: 10.1089/cpb.2008.0107

Ganzeboom, H. B.G. De Graaf, P. M., \& Treiman, D. J. (1992). A standard international socio-economic index of occupational status. Social Science Research, 21, 1-56, DOI: 10.1016/0049-089X(92)90017-B.

Gräsel, C., Fischer, F., \& Mandl, H. (2001). The use of additional information in problem-oriented learning environments. Learning Environments Research, 3, 287-305.

Guthrie, J. T., Wigfield, A., \& You, W. (2012). Instructional contexts for engagement and achievement in reading. In S. L. Christensen et al. (Eds.)., Handbook of research on student engagement (pp. 601-634). New York: Springer.

Guthrie, J. T., Wigfi eld, A., Barbosa, P., Perencevich, K. C., Taboada, A., Davis, M. H., et al. (2004). Increasing reading comprehension and engagement through Concept-Oriented Reading Instruction. Journal of Educational Psychology, 96 , 403-423. DOI: 10.1037/00220663.96.3.403

Hedges, L. V. \& Vevea, J. L. (1998). Fixed- and random effects models in metaanalysis. Psychological Methods, 3, 486-504. DOI: 10.1037/1082-989X.3.3.354

Hsu, Y.-C., \& Schwen, T. H. (2003). The effects of structural cues from multiple metaphors on computer users' information search performance. International Journal of Human-Computer Studies, 58, 39-55. DOI: 10.1016/S1071-5819(02)00125-8

Jacobsen, W. C., \& Forste, R. (2011). The wired generation: academic and social outcomes of electronic media use among university students. Cyberpsychology, Behavior, and Social Networking, 14, 275-280. DOI: 10.1089/cyber.2010.0135

Junco, R. (2012a). Too much face and not enough books: The relationship between multiple indices of Facebook use and academic performance. Computers in Human Behavior, 28, 187-198. DOI: 10.1016/j.chb.2011.08.026 
Junco, R. (2012b). The relationship between frequency of Facebook use, participation in Facebook activities, and student engagement. Computers \& Education, 58, 162-171. DOI: 10.1016/j.compedu.2011.08.004

Kirschner, P. A., \& Karpinski, A. C. (2010). Facebook ${ }^{\circledR}$ and academic performance. Computers in Human Behavior, 26, 1237-1245. DOI: 10.1016/j.chb.2010.03.024

Keil, F. C. \& Kominski, J. F. (2013). Missing links in middle school: Developing use of disciplinary relatedness in evaluating Internet search results. PLOS ONE, 8, 1-5. DOI: 10.1371/journal.pone.0067777

Lawless, K. A. \& Kulikowich, S. W. (1996). Understanding hypertext navigation through cluster analysis. Journal of Educational Computing Research, 14, 385-399. DOI: 10.2190/DVAP-DE23-3XMV-9MXH

Lawless, K. A., \& Schrader, P. G. (2008). Where do we go now? Understanding research on navigation in complex digital environments. In D. J. Leu \& J. Coiro (Eds.), Handbook of New Literacies (pp. 267-296). Hillsdale, NJ: Lawrence Erlbaum Associates.

Lee, Y.-H., \& Wu, J.-Y. (2013). The indirect effects of online social entertainment and information seeking activities on reading literacy. Computers \& Education, 67, 168-177. DOI: 10.1016/j.compedu.2013.03.001

Leu, D.J., Kinzer, C.K., Coiro, J., Cammack, D. (2004). Toward a theory of new literacies emerging from the Internet and other information and communication technologies. In R.B. Ruddell \& N. Unrau (Eds.), Theoretical models and processes of reading (5 $5^{\text {th }}$ ed.) (pp. 1568-1611). Newark, DE: International Reading Association.

McEneaney, J. E. (2001) Graphic and numerical methods to assess navigation in hypertext. International Journal of Human-Computer Studies, 55, 761-786. DOI:

10.1006/ijhc.2001.0505

Miniwatts Marketing Group (2012), Internet World Statistics, www.internetworldstats.com/stats.htm, accessed 23 January 2014. 
Naumann, J., Richter, T., Christmann, U. \& Groeben, N. (2008). Working memory capacity and reading skill moderate the effectiveness of strategy training in learning from hypertext. Learning and Individual Differences, 18, 197-213. DOI:

10.1016/j.lindif.2007.08.007

Naumann, J., Richter, T., Flender, J., Christmann, U., \& Groeben, N. (2007). Signaling in expository hypertexts compensates for deficits in reading skill. Journal of Educational Psychology, 99, 791-807. DOI: 10.1037/0022-0663.99.4.791

Niederhauser, D. S., Reynolds, R. E., Salmen, D. J., \& Skomolski, P. (2000). The influence of cognitive load on learning from hypertext. Journal of Educational Computing Research, 23, 237-255. DOI: 10.2190/81BG-RPDJ-9FA0-Q7PA

Rama, P. S., Black, R. W., van Es, E., \& Warschauer, M. (2012). Affordances for second language learning in World of Warcraft. ReCALL, 24, 322-338.

DOI:10.1017/S0958344012000171

OECD. (2011). PISA 2009 Results vol. VI: Student on line. Digital technologies and performance. Paris, France: OECD Publishing. DOI: 10.1787/9789264112995-en

OECD. (2012). PISA 2009 Technical report. Paris, France: OECD Publishing. DOI: $10.1787 / 9789264167872-e n$

OECD. (2013). OECD skills outlook 2013. First results from the survey of adult skills. Paris. France: OECD. DOI: 10.1787/9789264204256-en

OECD (2014). PISA 2012 Results: What Students Know and Can Do - Student Performance in Mathematics, Reading and Science (Volume I, Revised edition, February 2014) Paris. France: OECD Publishing. DOI: 10.1787/9789264201118-en

Pfost, M., Dörfler, T. \& Artelt, C. (2013). Students' extracurricular reading behavior and the development of vocabulary and reading comprehension. Learning and Individual Differences, 26, 89-102. DOI: 10.1016/j.lindif.2013.04.008 
Puntambekar, S., \& Stylianou, A. (2005). Designing navigation support in hypertext systems based on navigation patterns. Instructional Science, 33, 451-481. DOI:

$10.1007 / \mathrm{s} 11251-005-1276-5$

Puntambekar, S., Stylianou, A., \& Hübscher, R. (2003). Improving navigation and learning in hypertext environments with navigable concept maps. Human-Computer Interaction 18, 395-426. DOI: 10.1207/S15327051HCI1804_3

R Core Team (2012). R: A language and environment for statistical computing. $\mathrm{R}$ Foundation for Statistical Computing, Vienna, Austria. Retrieved January 14, 2014 from http://www.R-project.org/.

Richter, T., Naumann, J., \& Noller, S. (2003). LOGPAT: A semi-automatic way to analyze hypertext navigation behavior. Swiss Journal of Psychology, 62, 113-120. DOI: $10.1024 / / 1421-0185.62 .2 .113$

Richter, T., Naumann, J., Brunner, M., \& Christmann, U. (2005). Strategische Verarbeitung beim Lernen mit Text und Hypertext [Strategic processing in learning from text and hypertext]. Zeitschrift für Pädagogische Psychologie, 19, 5-22. DOI: 10.1024/10100652.19 .12 .5

Rouet, J.-F. (2006). The skills of document use. Mahwah, NJ: Erlbaum.

Salmerón, L., Cañas, J. J., Kintsch, W. J. \& Fajardo, I. (2005). Reading strategies and hypertext comprehension. Discourse Processes, 40, 171-191. DOI:

10.1207/s15326950dp4003_1

Salmerón, L., Cerdán, R., \& Naumann, J. (2015). How adolescents navigate Wikipedia to answer questions. Infancia y Aprendizaje: Journal for the Study of Education and Development, 38, 435-471. DOI: 10.1080/02103702.2015.1016750

Salmerón, L., \& García, V. (2011). Reading skills and children's navigation strategies in hypertext. Computers in Human Behaviour, 27, 1143-1151. DOI: 10.1016/j.chb.2010.12.008 
Salmerón, L., Kintsch, W. \& Cañas, J. J. (2006). Reading strategies and prior knowledge in learning from hypertext. Memory \& Cognition, 34, 1157-1171. DOI: 10.3758/BF03193262

Sana, F., Weston, T., \& Cepeda, N. J. (2013). Laptop multitasking hinders classroom learning for both users and nearby peers. Computers \& Education, 62, 24-31. DOI: 10.1016/j.compedu.2012.10.003

Schielzeth, H. (2010). Simple means to improve the interpretability of regression coefficients. Methods in Ecology and Evolution, 1, 103-113. DOI: 1/j.2041210X.2010.00012.x

Stanovich, K. E. (1986). Matthew effects in reading: Some consequences of individual differences in the acquisition of literacy. Reading Research Quarterly, 21, 360-407.

Stanovich, K. E. (2000). Progress in understanding reading. Scientific foundations and new frontiers. New York, NY: Guilford Press.

Su, Y., \& Klein, J. D. (2006). Effects of navigation tools and computer confidence on performance and attitudes in a hypermedia learning environment. Journal of Educational Multimedia and Hypermedia, 15, 87-106.

Thorne, S. L., \& Black, R. (2007). Language and literacy development in computer mediated contexts and communities. Annual Review of Applied Linguistics, 27, 133-160. DOI: $10.1017 / \mathrm{S} 0267190508070074$

Viechtbauer, W. (2010). Conducting meta-analyses in R with the metafor package. Journal of Statistical Software, 36 (3), 1-48.

Wigfield, A., Guthrie, J. T., Perencevich, K. C., Taboada, A., Klauda, S. L., McRae, A., et al. (2008). The role of reading engagement in mediating effects of reading comprehension instruction on reading outcomes. Psychology in the Schools, 45, 432-445. DOI: 10.1002/pits.20307

Wu, M., Adams, R., \& Haldane, S. (2007). ACER Conquest 2.0. Generalized item response modeling software [Computer Program]. Melbourne: ACER Press. 
Zhang, X, de Pablos, P. O., \& Xu, Q. (2014). Culture effects on the knowledge sharing in multi-national virtual classes: A mixed method. Computers in Human Behavior , 31, 491498. DOI: 10.1016/j.chb.2013.04.021

Zhang, X., de Pablos, P., \& Zhou., Z. (2013). Effect of Knowledge Sharing Visibility on incentive-based relationship in Electronic Knowledge Management Systems: An Empirical Investigation. Computers in Human Behavior, 29, 307-313. DOI: 10.1016/j.chb.2012.01.029

Zhang, X., Vogel, D., \& Zhou, Z. (2012). Effects of Information Technologies, Department Characteristics and Individual Roles on Improving Knowledge Sharing Visibility: A Qualitative Case Study. Behaviour \& Information Technology, 31, 1117-1131. DOI: 10.1080/0144929X.2012.687770 
Table 1

Descriptive statistics and correlations of all variables in the study (complete sample)

Correlations

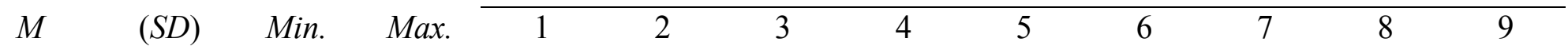

\begin{tabular}{|c|c|c|c|c|c|c|c|c|c|c|c|c|c|}
\hline 1. Performance ${ }^{a, d}$ & 0.69 & $(0.46)$ & 0.00 & 1.00 & & & & & & & & & \\
\hline 2. Information Access ${ }^{a}$ & 4.32 & $(5.31)$ & 0.00 & 88.00 & $.15^{\mathrm{e}}$ & & & & & & & & \\
\hline 3. Access Demands ${ }^{b}$ & 3.62 & $(3.42)$ & 1.00 & 14.00 & $.05^{\mathrm{e}}$ & .63 & & & & & & & \\
\hline 4. Information Engagement & 0.71 & $(1.37)$ & -5.23 & 4.64 & $.06^{\mathrm{e}}$ & .03 & .01 & & & & & & \\
\hline 5. Social Engagement & -0.04 & $(0.81)$ & -2.01 & 2.14 & $-.01^{\mathrm{e}}$ & .00 & .01 & .34 & & & & & \\
\hline 6. Print reading skill & 506.27 & $(99.05)$ & 0.00 & 884.66 & $.29^{\mathrm{e}}$ & .10 & .03 & .18 & -.04 & & & & \\
\hline 7. Use of ICT at home & 0.03 & $(0.95)$ & -4.19 & 1.41 & $.04^{\mathrm{e}}$ & .00 & .01 & .19 & .33 & .07 & & & \\
\hline 8. Use of ICT at school & 0.10 & $(0.94)$ & -2.79 & 1.80 & $.01^{\mathrm{e}}$ & -.01 & .01 & .06 & .12 & .02 & .24 & & \\
\hline 9. Gender ${ }^{\mathrm{c}}$ & 0.51 & $(0.50)$ & 0.00 & 1.00 & $.05^{\mathrm{f}}$ & $.00^{\mathrm{e}}$ & $.00^{\mathrm{e}}$ & $-.02^{\mathrm{e}}$ & $-.05^{\mathrm{e}}$ & $.17^{\mathrm{e}}$ & $-.09^{\mathrm{e}}$ & $.01^{\mathrm{e}}$ & \\
\hline 10. SES & 49.68 & (16.13) & 16.00 & 90.00 & .12 & .04 & .02 & .12 & .05 & .29 & .24 & .06 & -.02 \\
\hline
\end{tabular}

Note. Correlations are Pearson product-moment correlations, unless noted otherwise. Means and standard deviations are unweighted. $N=29,395$ students.

${ }^{\mathrm{a}} I=524,771$ task responses. ${ }^{\mathrm{b}} K=29$ tasks. ${ }^{\mathrm{c}}$ Dummy-coded, Boys $=0$, Girls $=1 .{ }^{\mathrm{d}}$ Dichotomous, $1=$ credit, $0=$ no credit. ${ }^{\mathrm{e}}$ Point-biserial correlation. ${ }^{\mathrm{f}}$ Phi-coefficient. 
Table 2

Prediction of Information access behavior: Effects of access demands and Information related engagement

\begin{tabular}{|c|c|c|c|c|c|c|c|c|c|c|c|}
\hline \multirow[b]{2}{*}{ Country } & \multirow{2}{*}{$\begin{array}{r}\text { Intercept } \\
\quad b(S E)\end{array}$} & \multicolumn{2}{|c|}{ Access demands } & \multicolumn{2}{|c|}{$\begin{array}{c}\text { Information } \\
\text { engagement }^{\text {b }}\end{array}$} & \multicolumn{2}{|c|}{$\begin{array}{c}\text { Information } \\
\text { engagement } \times \\
\text { access demands }\end{array}$} & \multicolumn{2}{|c|}{$\begin{array}{c}\text { Social } \\
\text { engagement }^{\mathrm{b}}\end{array}$} & \multicolumn{2}{|c|}{$\begin{array}{c}\text { Social } \\
\text { engagement } \times \\
\text { access demands }^{\mathrm{c}}\end{array}$} \\
\hline & & $b(S E)$ & $\begin{array}{l}\text { Pred. } \\
\text { Diff. }^{d}\end{array}$ & $b(S E)$ & $\begin{array}{l}\text { Pred. } \\
\text { Diff. }^{\text {e }}\end{array}$ & $b(S E)$ & $\begin{array}{l}\text { Pred. } \\
\text { Diff. }^{f}\end{array}$ & $b(S E)$ & $\begin{array}{l}\text { Pred. } \\
\text { Diff. }^{\text {g }}\end{array}$ & $b(S E)$ & $\begin{array}{l}\text { Pred. } \\
\text { Diff. }^{\text {h }}\end{array}$ \\
\hline AUS & $4.39(0.46)$ & $3.49(0.44)^{\mathrm{a}}$ & 6.99 & $0.12(0.02)^{\mathrm{a}}$ & 0.24 & $0.29(0.01)^{\mathrm{a}}$ & 0.57 & $-0.01(0.02)$ & -0.02 & $-0.10(0.01)^{\mathrm{a}}$ & -0.20 \\
\hline AUT & $3.68(0.38)$ & $2.57(0.34)^{\mathrm{a}}$ & 5.14 & $0.05(0.02)$ & 0.11 & $0.20(0.02)^{\mathrm{a}}$ & 0.40 & $0.01(0.02)$ & 0.02 & $-0.07(0.02)^{\mathrm{a}}$ & -0.15 \\
\hline BEL & $4.16(0.43)$ & $3.13(0.40)^{\mathrm{a}}$ & 6.27 & $0.08(0.02)^{\mathrm{a}}$ & 0.16 & $0.13(0.01)^{\mathrm{a}}$ & 0.26 & $0.00(0.02)$ & 0.00 & $-0.07(0.01)^{\mathrm{a}}$ & -0.14 \\
\hline $\mathrm{CHL}$ & $3.99(0.47)$ & $2.65(0.41)^{\mathrm{a}}$ & 5.30 & $0.03(0.04)$ & 0.06 & $0.17(0.02)^{\mathrm{a}}$ & 0.35 & $0.01(0.04)$ & 0.01 & $0.09(0.02)$ & 0.18 \\
\hline SWE & $4.34(0.44)$ & $3.22(0.41)^{\mathrm{a}}$ & 6.44 & $0.10(0.03)^{\mathrm{a}}$ & 0.20 & $0.17(0.02)^{\mathrm{a}}$ & 0.33 & $-0.02(0.03)$ & -0.03 & $-0.08(0.02)^{\mathrm{a}}$ & -0.17 \\
\hline DNK & $3.90(0.40)$ & $2.85(0.37)^{\mathrm{a}}$ & 5.70 & $0.04(0.03)$ & 0.08 & $0.03(0.02)$ & 0.06 & $-0.01(0.03)$ & -0.01 & $-0.03(0.02)$ & -0.06 \\
\hline ESP & $4.00(0.43)$ & $2.81(0.40)^{\mathrm{a}}$ & 5.62 & $0.07(0.03)$ & 0.14 & $0.15(0.02)^{\mathrm{a}}$ & 0.30 & $-0.02(0.03)$ & -0.03 & $0.06(0.02)^{\mathrm{a}}$ & 0.12 \\
\hline POL & $3.87(0.42)$ & $2.77(0.35)^{\mathrm{a}}$ & 5.54 & $0.10(0.03)^{\mathrm{a}}$ & 0.20 & $0.24(0.02)^{\mathrm{a}}$ & 0.47 & $0.02(0.03)$ & 0.04 & $0.04(0.02)$ & 0.07 \\
\hline HKG & $5.36(0.57)$ & $4.42(0.52)^{\mathrm{a}}$ & 8.83 & $0.06(0.04)$ & 0.13 & $0.19(0.03)^{\mathrm{a}}$ & 0.37 & $0.02(0.04)$ & 0.04 & $-0.03(0.03)$ & -0.06 \\
\hline HUN & $3.73(0.39)$ & $2.44(0.35)^{\mathrm{a}}$ & 4.88 & $0.10(0.03)^{\mathrm{a}}$ & 0.20 & $0.27(0.02)^{\mathrm{a}}$ & 0.53 & $0.00(0.03)$ & 0.01 & $-0.06(0.02)^{\mathrm{a}}$ & -0.11 \\
\hline IRL & $4.08(0.38)$ & $3.09(0.36)^{\mathrm{a}}$ & 6.18 & $0.09(0.03)^{\mathrm{a}}$ & 0.18 & $0.17(0.02)^{\mathrm{a}}$ & 0.34 & $0.02(0.03)$ & 0.04 & $-0.01(0.02)$ & -0.02 \\
\hline ISL & $4.32(0.49)$ & $2.98(0.47)^{\mathrm{a}}$ & 5.95 & $0.07(0.04)$ & 0.13 & $0.09(0.03)^{\mathrm{a}}$ & 0.18 & $0.02(0.04)$ & 0.04 & $-0.08(0.03)^{\mathrm{a}}$ & -0.15 \\
\hline JPN & $5.09(0.53)$ & $3.98(0.49)^{\mathrm{a}}$ & 7.96 & $0.03(0.04)$ & 0.06 & $0.12(0.03)^{\mathrm{a}}$ & 0.24 & $0.07(0.04)$ & 0.15 & $0.08(0.03)^{\mathrm{a}}$ & 0.16 \\
\hline KOR & $5.39(0.57)$ & $4.37(0.55)^{\mathrm{a}}$ & 8.73 & $0.05(0.03)$ & 0.11 & $0.11(0.02)^{\mathrm{a}}$ & 0.22 & $-0.05(0.03)$ & -0.10 & $-0.16(0.02)^{\mathrm{a}}$ & -0.31 \\
\hline MAC & $5.53(0.67)$ & $4.13(0.61)^{a}$ & 8.25 & $0.09(0.03)^{\mathrm{a}}$ & 0.18 & $0.19(0.02)^{\mathrm{a}}$ & 0.39 & $0.03(0.03)$ & 0.05 & $-0.04(0.02)$ & -0.08 \\
\hline NOR & $3.97(0.42)$ & $2.82(0.40)^{\mathrm{a}}$ & 5.64 & $0.06(0.03)$ & 0.12 & $0.04(0.02)^{\mathrm{a}}$ & 0.09 & $-0.03(0.03)$ & -0.07 & $-0.08(0.02)^{\mathrm{a}}$ & -0.17 \\
\hline NZL & $4.52(0.46)$ & $3.67(0.44)^{\mathrm{a}}$ & 7.35 & $0.17(0.03)^{\mathrm{a}}$ & 0.35 & $0.28(0.02)^{\mathrm{a}}$ & 0.56 & $0.07(0.03)$ & 0.14 & $0.06(0.02)$ & 0.12 \\
\hline
\end{tabular}

Note. Effect sizes for insignificant effects are printed in italics.

${ }^{\mathrm{a}} p<.05$ (family-wise), nominal $\alpha=0.01{ }^{\mathrm{b}}{ }^{\text {Two-tailed tests. }}{ }^{\mathrm{c}}$ one-tailed tests. ${ }^{\mathrm{d}}$ Effect size estimate: Predicted difference in the number of visits to task-relevant pages between a task high $(+1 S D)$ and low $(-1 S D)$ in access demands. ${ }^{\mathrm{e}}$ Effect size estimate: Predicted difference in the number of visits to task-relevant pages between a student high $(+1 S D)$ and low $(-1 S D)$ in information online reading engagement. ${ }^{\mathrm{f}}$ Effect size estimate. Predicted difference in the effect of information online reading

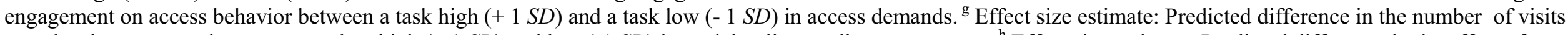
to task-relevant pages between a student high $(+1 S D)$ and low $(-1 S D)$ in social online reading engagement. ${ }^{\text {h }}$ Effect size estimate. Predicted difference in the effect of social online reading engagement on access behavior between a task high $(+1 S D)$ and a task low $(-1 S D)$ in access demands. 
Table 3

Prediction of information access: Simple slopes of information and social online reading engagement

\begin{tabular}{|c|c|c|c|c|c|c|c|c|}
\hline \multirow[b]{2}{*}{ Country } & \multicolumn{2}{|c|}{$\begin{array}{c}\text { Information } \\
\text { engagement at } \\
\text { access demands }=+1 S D\end{array}$} & \multicolumn{2}{|c|}{$\begin{array}{c}\text { Information } \\
\text { engagement at } \\
\text { access demands }=-1 S D\end{array}$} & \multicolumn{2}{|c|}{$\begin{array}{c}\text { Social } \\
\text { engagement at } \\
\text { access demands }=+1 S D\end{array}$} & \multicolumn{2}{|c|}{$\begin{array}{c}\text { Social } \\
\text { engagement at } \\
\text { access demands }=-1 S D\end{array}$} \\
\hline & $b(S E)$ & $\begin{array}{l}\text { Pred. } \\
\text { Diff. }^{b}\end{array}$ & $b(S E)$ & $\begin{array}{l}\text { Pred. } \\
\text { Diff. }^{c}\end{array}$ & $b(S E)$ & $\begin{array}{l}\text { Pred. } \\
\text { Diff. }^{d}\end{array}$ & $b(S E)$ & $\begin{array}{l}\text { Pred. } \\
\text { Diff. }^{e}\end{array}$ \\
\hline AUS & $0.41(0.03)^{\mathrm{a}}$ & 0.82 & $-0.16(0.03)^{\mathrm{a}}$ & -0.33 & $-0.11(0.03)^{\mathrm{a}}$ & -0.22 & $0.09(0.03)^{\mathrm{a}}$ & 0.17 \\
\hline AUT & $0.25(0.03)^{\mathrm{a}}$ & 0.51 & $-0.15(0.03)^{\mathrm{a}}$ & -0.29 & $-0.06(0.03)^{a}$ & -0.12 & $0.08(0.03)^{\mathrm{a}}$ & 0.17 \\
\hline BEL & $0.21(0.02)^{\mathrm{a}}$ & 0.42 & $-0.05(0.02)^{\mathrm{a}}$ & -0.10 & $-0.07(0.02)^{\mathrm{a}}$ & -0.14 & $0.07(0.02)^{\mathrm{a}}$ & 0.14 \\
\hline CHL & $0.20(0.05)^{\mathrm{a}}$ & 0.41 & $-0.14(0.05)^{\mathrm{a}}$ & -0.29 & $0.10(0.05)$ & 0.19 & $-0.09(0.05)$ & -0.17 \\
\hline SWE & $0.27(0.03)^{\mathrm{a}}$ & 0.54 & $-0.06(0.03)^{\mathrm{a}}$ & -0.13 & $-0.10(0.03)^{\mathrm{a}}$ & -0.20 & $0.07(0.03)^{\mathrm{a}}$ & 0.13 \\
\hline DNK & $0.07(0.04)^{\mathrm{a}}$ & 0.14 & $0.01(0.04)$ & 0.02 & $-0.04(0.04)$ & -0.07 & $0.02(0.04)$ & 0.05 \\
\hline ESP & $0.22(0.04)^{\mathrm{a}}$ & 0.44 & $-0.08(0.04)^{\mathrm{a}}$ & -0.16 & $0.05(0.04)$ & 0.09 & $-0.08(0.04)$ & -0.15 \\
\hline POL & $0.34(0.04)^{\mathrm{a}}$ & 0.67 & $-0.14(0.04)^{\mathrm{a}}$ & -0.28 & $0.05(0.04)$ & 0.11 & $-0.02(0.04)$ & -0.03 \\
\hline HKG & $0.25(0.05)^{\mathrm{a}}$ & 0.50 & $-0.12(0.05)^{\mathrm{a}}$ & -0.25 & $-0.01(0.05)$ & -0.03 & $0.05(0.05)$ & 0.10 \\
\hline HUN & $0.36(0.04)^{\mathrm{a}}$ & 0.73 & $-0.17(0.04)^{\mathrm{a}}$ & -0.33 & $-0.05(0.04)$ & -0.11 & $0.06(0.04)^{\mathrm{a}}$ & 0.12 \\
\hline IRL & $0.26(0.04)^{\mathrm{a}}$ & 0.52 & $-0.08(0.04)^{\mathrm{a}}$ & -0.16 & $0.01(0.04)$ & 0.02 & $0.03(0.04)$ & 0.07 \\
\hline ISL & $0.16(0.05)^{\mathrm{a}}$ & 0.31 & $-0.02(0.05)$ & -0.05 & $-0.06(0.04)$ & -0.11 & $0.10(0.04)^{\mathrm{a}}$ & 0.20 \\
\hline JPN & $0.15(0.05)^{\mathrm{a}}$ & 0.30 & $-0.09(0.05)^{\mathrm{a}}$ & -0.18 & $0.15(0.05)$ & 0.30 & $-0.01(0.05)$ & -0.01 \\
\hline KOR & $0.17(0.04)^{\mathrm{a}}$ & 0.33 & $-0.06(0.04)$ & -0.12 & $-0.21(0.04)^{\mathrm{a}}$ & -0.41 & $0.11(0.04)^{\mathrm{a}}$ & 0.21 \\
\hline MAC & $0.28(0.04)^{\mathrm{a}}$ & 0.56 & $-0.11(0.04)^{\mathrm{a}}$ & -0.21 & $-0.01(0.04)$ & -0.03 & $0.07(0.04)^{\mathrm{a}}$ & 0.14 \\
\hline NOR & $0.10(0.03)^{\mathrm{a}}$ & 0.21 & $0.02(0.03)$ & 0.04 & $-0.12(0.03)^{\mathrm{a}}$ & -0.23 & $0.05(0.03)^{\mathrm{a}}$ & 0.10 \\
\hline NZL & $0.45(0.04)^{\mathrm{a}}$ & 0.90 & $-0.10(0.03)^{\mathrm{a}}$ & -0.21 & $0.13(0.03)$ & 0.26 & $0.01(0.03)$ & 0.02 \\
\hline
\end{tabular}

Note. Effect sizes for insignificant effects are printed in italics.

${ }^{\mathrm{a}} p<.05$ (one-tailed tests). ${ }^{\mathrm{b}}$ Effect size estimate: Predicted difference in the umber of visits to task-relevant pages between a student high (+ $\left.1 \mathrm{SD}\right)$ and a student low (-1 SD) in information online reading engagement within a high navigation demands task. ${ }^{\mathrm{c}}$ Effect size estimate: Predicted difference in the umber of visits to task-relevant pages between a student high $(+1 \mathrm{SD})$ and a student low $(-1 \mathrm{SD})$ in information online reading engagement within a low navigation demands task.

$\left.{ }^{\mathrm{d} E f f e c t ~ s i z e: ~ e s t i m a t e: ~ P r e d i c t e d ~ d i f f e r e n c e ~ i n ~ t h e ~ u m b e r ~ o f ~ v i s i t s ~ t o ~ t a s k-r e l e v a n t ~ p a g e s ~ b e t w e e n ~ a ~ s t u d e n t ~ h i g h ~(+~} 1 \mathrm{SD}\right)$ and a student low (-1 SD) in social online reading engagement within a high navigation demands task. ${ }^{e}$ Effect size estimate: Predicted difference in the umber of visits to task-relevant pages between a student high (+ 1 SD) and a student low (- 1 SD) in social online reading engagement within a low navigation demands task. 
Table 4

Prediction of task performance: Effects of reading skill, access demands, and access behavior

\begin{tabular}{|c|c|c|c|c|c|c|c|c|c|}
\hline \multirow[b]{2}{*}{ Country } & \multirow{2}{*}{$\begin{array}{l}\text { Intercept } \\
\quad b(S E)\end{array}$} & \multicolumn{2}{|c|}{ Reading skill } & \multicolumn{2}{|c|}{ Access demands } & \multicolumn{2}{|c|}{ Access behavior } & \multicolumn{2}{|c|}{$\begin{array}{l}\text { Access demands } \times \\
\text { access behavior }\end{array}$} \\
\hline & & $b(S E)$ & $\begin{array}{l}\text { Pred. } \\
\text { Diff. }^{b}\end{array}$ & $b(S E)$ & $\begin{array}{l}\text { Pred. } \\
\text { Diff. }\end{array}$ & $b(S E)$ & $\begin{array}{l}\text { Pred. } \\
\text { Diff. }^{d}\end{array}$ & $b(S E)$ & $\begin{array}{l}\text { Pred. } \\
\text { Diff. }\end{array}$ \\
\hline AUS & $1.41(0.22)$ & $0.85(0.02)^{\mathrm{a}}$ & 0.27 & $-0.08(0.21)$ & -0.02 & $0.34(0.04)^{\mathrm{a}}$ & 0.11 & $0.17(0.03)^{\mathrm{a}}$ & 0.12 \\
\hline AUT & $0.42(0.23)$ & $0.70(0.02)^{\mathrm{a}}$ & 0.33 & $-0.16(0.20)$ & -0.08 & $0.37(0.03)^{\mathrm{a}}$ & 0.18 & $0.20(0.02)^{\mathrm{a}}$ & 0.19 \\
\hline BEL & $1.15(0.22)$ & $0.80(0.02)^{\mathrm{a}}$ & 0.29 & $-0.15(0.20)$ & -0.06 & $0.34(0.03)^{\mathrm{a}}$ & 0.12 & $0.12(0.02)^{\mathrm{a}}$ & 0.11 \\
\hline CHL & $0.48(0.21)$ & $0.66(0.03)^{\mathrm{a}}$ & 0.30 & $-0.25(0.19)$ & -0.12 & $0.34(0.04)^{\mathrm{a}}$ & 0.16 & $0.11(0.02)^{\mathrm{a}}$ & 0.12 \\
\hline SWE & $1.17(0.21)$ & $0.81(0.02)^{\mathrm{a}}$ & 0.29 & $-0.28(0.20)$ & -0.10 & $0.44(0.04)^{\mathrm{a}}$ & 0.16 & $0.14(0.03)^{\mathrm{a}}$ & 0.14 \\
\hline DNK & $0.68(0.23)$ & $0.77(0.03)^{\mathrm{a}}$ & 0.33 & $-0.22(0.21)$ & -0.10 & $0.33(0.05)^{\mathrm{a}}$ & 0.15 & $0.22(0.03)^{\mathrm{a}}$ & 0.21 \\
\hline ESP & $0.74(0.20)$ & $0.72(0.02)^{\mathrm{a}}$ & 0.31 & $-0.22(0.18)$ & -0.10 & $0.34(0.04)^{\mathrm{a}}$ & 0.15 & $0.15(0.03)^{\mathrm{a}}$ & 0.15 \\
\hline POL & $0.71(0.24)$ & $0.76(0.02)^{\mathrm{a}}$ & 0.32 & $-0.21(0.20)$ & -0.09 & $0.49(0.04)^{\mathrm{a}}$ & 0.21 & $0.07(0.03)^{\mathrm{a}}$ & 0.09 \\
\hline HKG & $1.16(0.23)$ & $0.62(0.03)^{\mathrm{a}}$ & 0.22 & $-0.16(0.21)$ & -0.06 & $0.37(0.05)^{\mathrm{a}}$ & 0.13 & $0.19(0.03)^{\mathrm{a}}$ & 0.16 \\
\hline HUN & $0.61(0.21)$ & $0.80(0.03)^{\mathrm{a}}$ & 0.35 & $-0.39(0.19)$ & -0.17 & $0.52(0.04)^{\mathrm{a}}$ & 0.23 & $0.12(0.03)^{\mathrm{a}}$ & 0.15 \\
\hline IRL & $1.06(0.23)$ & $0.68(0.03)^{\mathrm{a}}$ & 0.26 & $-0.18(0.21)$ & -0.07 & $0.31(0.05)^{\mathrm{a}}$ & 0.12 & $0.14(0.03)^{\mathrm{a}}$ & 0.12 \\
\hline ISL & $1.08(0.22)$ & $0.77(0.04)^{\mathrm{a}}$ & 0.29 & $-0.05(0.21)$ & -0.02 & $0.25(0.05)^{\mathrm{a}}$ & 0.09 & $0.22(0.04)^{\mathrm{a}}$ & 0.17 \\
\hline JPN & $1.49(0.22)$ & $0.42(0.03)^{\mathrm{a}}$ & 0.13 & $-0.16(0.20)$ & -0.05 & $0.38(0.06)^{\mathrm{a}}$ & 0.11 & $0.11(0.04)^{\mathrm{a}}$ & 0.09 \\
\hline KOR & $1.75(0.24)$ & $0.58(0.03)^{\mathrm{a}}$ & 0.15 & $0.00(0.22)$ & 0.00 & $0.19(0.05)^{\mathrm{a}}$ & 0.05 & $0.17(0.04)^{\mathrm{a}}$ & 0.09 \\
\hline MAC & $0.86(0.25)$ & $0.57(0.02)^{\mathrm{a}}$ & 0.23 & $-0.04(0.23)$ & -0.02 & $0.20(0.03)^{\mathrm{a}}$ & 0.09 & $0.20(0.02)^{\mathrm{a}}$ & 0.17 \\
\hline NOR & $0.92(0.23)$ & $0.76(0.02)^{\mathrm{a}}$ & 0.30 & $-0.22(0.22)$ & -0.09 & $0.36(0.04)^{\mathrm{a}}$ & 0.14 & $0.24(0.03)^{\mathrm{a}}$ & 0.22 \\
\hline NZL & $1.49(0.22)$ & $0.84(0.03)^{\mathrm{a}}$ & 0.26 & $-0.09(0.21)$ & -0.03 & $0.29(0.04)^{\mathrm{a}}$ & 0.09 & $0.20(0.03)^{\mathrm{a}}$ & 0.13 \\
\hline
\end{tabular}

Note. Effect sizes for insignificant effects are printed in italics.

${ }^{\mathrm{a}} p<.05$ (family-wise), nominal $\alpha=0.0125$, one-tailed. ${ }^{\mathrm{b}}$ Effect size estimate: Predicted difference in the probability of correctly solving a task between a student high $(+1 \mathrm{SD})$ and a student low $(-1 \mathrm{SD})$ in reading skill. ${ }^{\mathrm{c}}$ Effect size estimate: Predicted difference in probability of correctly solving a task between a task high $(+1$ $S D)$ and low (-1 SD) in access demands. ${ }^{\mathrm{d}}$ Effect size estimate: Predicted difference in the probability of correctly solving a task between a making many $(+1$ SD) and a student making few (- 1 SD) visits and re-visits to task relevant pages. ${ }^{\mathrm{e}}$ Effect size estimate: Predicted difference in the effect of access behvior on the probability of correctly solving a digital reading task between a task high $(+1 S D)$ and a task low $(-1 S D)$ in access demands. 
Table 5

Prediction of task performance: Simple slopes

\begin{tabular}{|c|c|c|c|c|}
\hline \multirow[b]{2}{*}{ Country } & \multicolumn{2}{|c|}{$\begin{array}{l}\text { Access behavior at } \\
\text { access demands } \\
=+1 \mathrm{SD}\end{array}$} & \multicolumn{2}{|c|}{$\begin{array}{l}\text { Access behavior at } \\
\text { access demands }\end{array}$} \\
\hline & $b(S E)$ & Pred. Diff. & $b(S E)$ & Pred. Diff. ${ }^{\mathrm{c}}$ \\
\hline AUS & $0.51(0.03)^{\mathrm{a}}$ & 0.17 & $0.18(0.06)$ & 0.05 \\
\hline AUT & $0.56(0.02)^{\mathrm{a}}$ & 0.27 & $0.17(0.05)$ & 0.08 \\
\hline BEL & $0.46(0.02)^{\mathrm{a}}$ & 0.18 & $0.22(0.05)$ & 0.07 \\
\hline CHL & $0.44(0.03)^{\mathrm{a}}$ & 0.22 & $0.23(0.06)$ & 0.10 \\
\hline SWE & $0.58(0.03)^{\mathrm{a}}$ & 0.24 & $0.31(0.06)$ & 0.09 \\
\hline DNK & $0.56(0.04)^{\mathrm{a}}$ & 0.26 & $0.11(0.08)$ & 0.04 \\
\hline ESP & $0.49(0.03)^{\mathrm{a}}$ & 0.23 & $0.19(0.06)$ & 0.08 \\
\hline POL & $0.56(0.03)^{\mathrm{a}}$ & 0.26 & $0.43(0.06)$ & 0.17 \\
\hline $\mathrm{HKG}$ & $0.56(0.04)^{\mathrm{a}}$ & 0.22 & $0.18(0.08)$ & 0.06 \\
\hline HUN & $0.63(0.03)^{\mathrm{a}}$ & 0.30 & $0.40(0.06)$ & 0.16 \\
\hline IRL & $0.45(0.03)^{\mathrm{a}}$ & 0.19 & $0.18(0.07)$ & 0.06 \\
\hline ISL & $0.47(0.04)^{\mathrm{a}}$ & 0.18 & $0.03(0.09)$ & 0.01 \\
\hline JPN & $0.49(0.04)^{\mathrm{a}}$ & 0.16 & $0.27(0.09)$ & 0.07 \\
\hline KOR & $0.37(0.04)^{\mathrm{a}}$ & 0.09 & $0.02(0.08)$ & 0.00 \\
\hline MAC & $0.40(0.02)^{\mathrm{a}}$ & 0.17 & $0.00(0.05)$ & 0.00 \\
\hline NOR & $0.60(0.03)^{\mathrm{a}}$ & 0.26 & $0.11(0.06)$ & 0.04 \\
\hline NZL & $0.49(0.03)^{\mathrm{a}}$ & 0.16 & $0.09(0.07)$ & 0.03 \\
\hline
\end{tabular}

Note. Effect sizes for insignificant effects are printed in italics.

${ }^{\mathrm{a}} p<.05$ (one-tailed tests). ${ }^{\mathrm{b}}$ Effect size estimate: Predicted difference in the probability of correctly solving a task for a student low in the number of visits to task-relevant pages $(-1 S D)$ and a student high in the number of visits to task-relevant pages $(+1 S D)$ for a task high in navigation demands. ${ }^{c}$ Effect size estimate: Predicted difference in the probability of correctly solving a task for a student showing low in the number of visits to task-relevant pages $(-1 S D)$ and a student high in the number of visits to task-relevant pages $(+1 S D)$ for a task low in navigation demands. 


\section{Figure captions}

Figure 1: Model of online reading engagement, adapted after Guthrie et al. (2012; p. 604). Variables in the model addressed in the present research are shaded grey, associations addressed in the present research are depicted as solid lines. Variables the model that not addressed in the present research are shaded white, associations not addressed in the present research are depicted as dashed lines. Circles with an $\times$ indicate interaction effects

Figure 2: Screenshot of the prompting page of a sample Digital Reading Assessment task. This task is high in access demands, and requires the student to visit eight different pages to complete the task. The number of relevant pages in this task is 15 . Refer to http://erasq.acer.edu.au/ for an operational version of this and other released Digital Reading Assessment tasks.

Figure 3: (a) Distribution of simple slopes for Information engagement (white) and Social engagement (grey), each for tasks high (left) and low (right) in access demands. (b) Metaanalytical simple slopes for Information engagement (dashed lines) and Social engagement (solid lines) in predicting access behavior conditional on high access demands tasks (upper lines) and low access demands tasks (lower lines). (c) Distribution of simple slopes for access behavior (relevant page visits) in tasks high (left) and low (right) in access demands. (d) Meta-analytical simple slopes for access behavior (relevant page visits) in predicting access behavior conditional on high access demands tasks (straight line) and low access demands tasks (dashed line). See text for significance tests of slopes. 


\section{Student level}

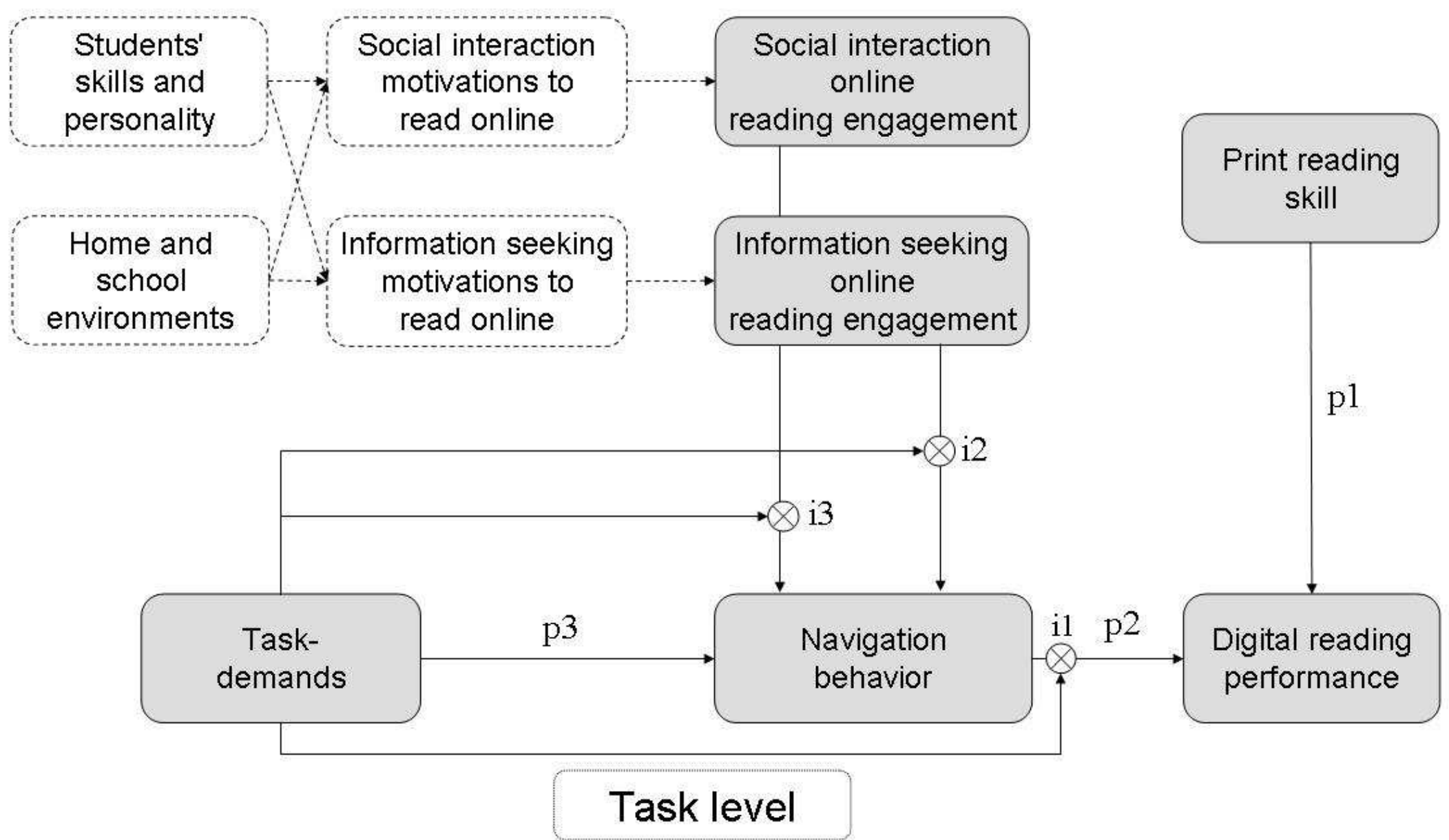




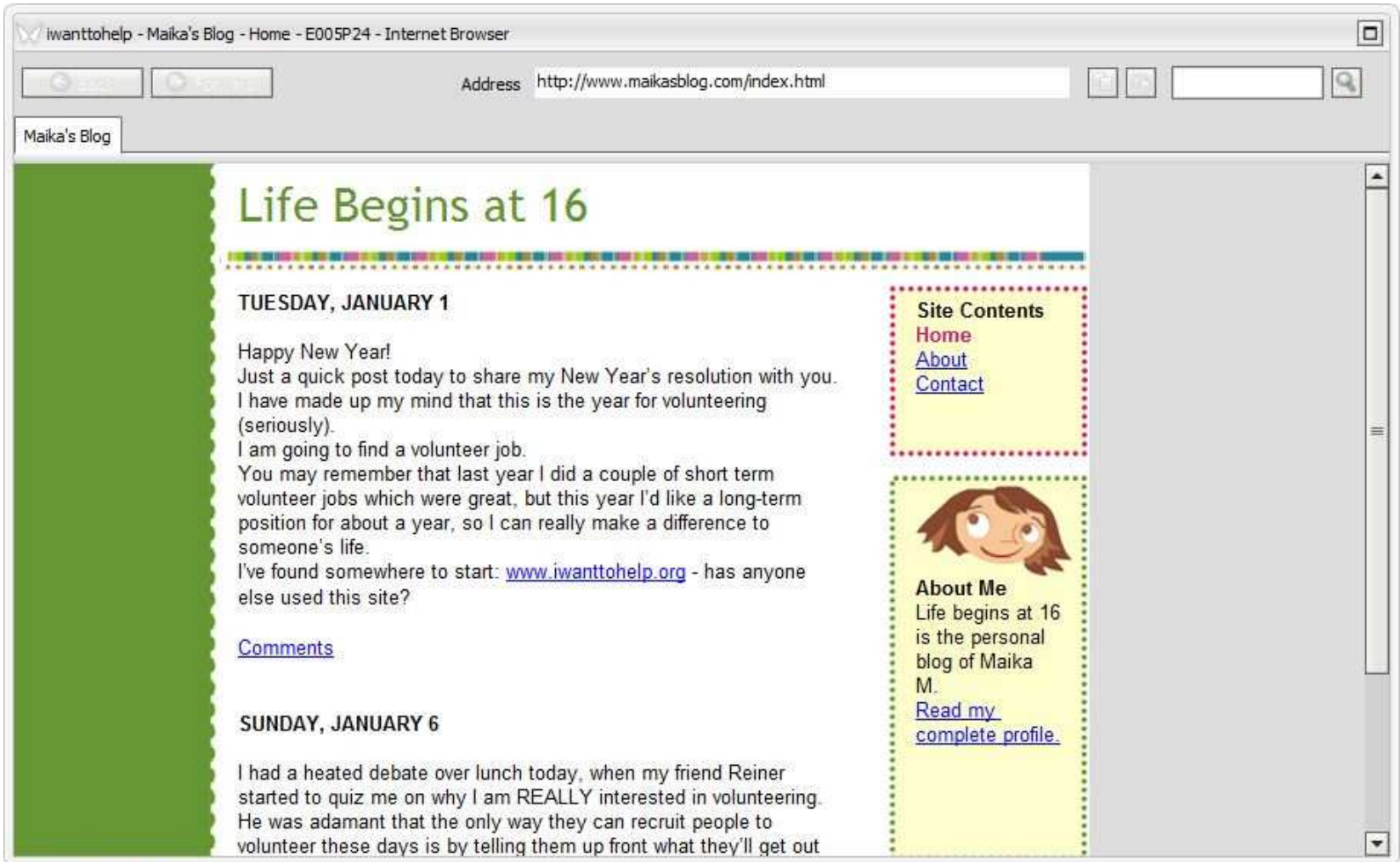

IWANTTOHELP: Task 4

Read Maika's blog for January 1. Go to the iwanttohelp site and find an opportunity for Maika. Use the e-mail button on the "Opportunity Details" page to tell Maika about this opportunity. Explain in the e-mail why you think the opportunity is suitable for her. Then send your e-mail by clicking on the "Send" button. 
(a) Distribution of simple slopes

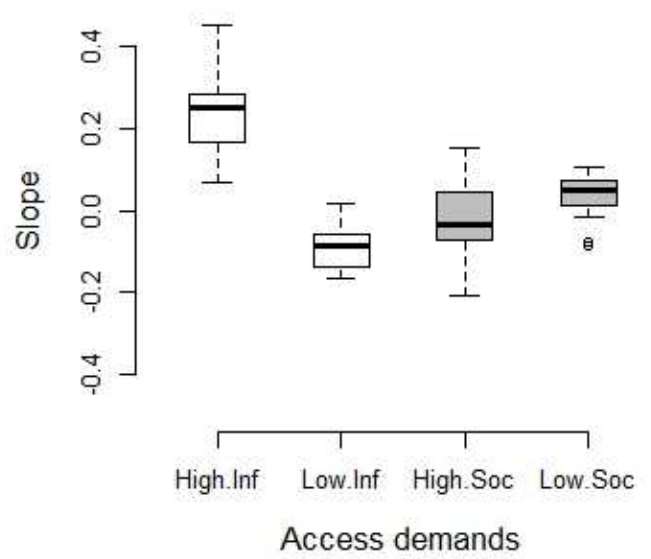

(c) Distribution of simple slopes

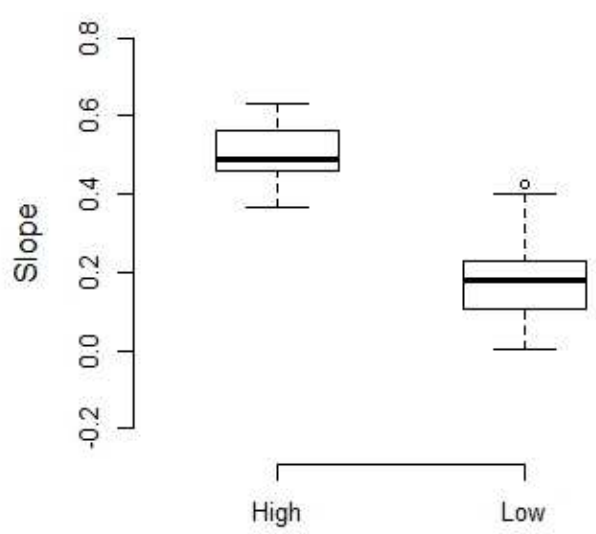

Access demands (b) Meta-analytical simple slopes

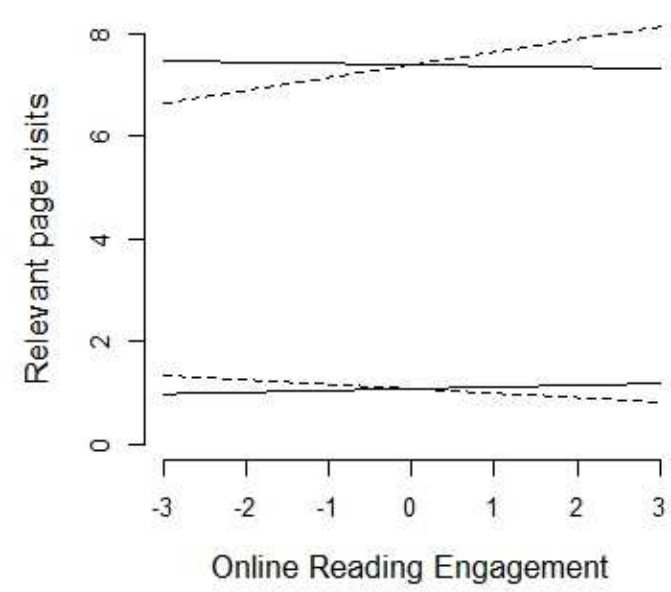

(d) Meta-analytical simple slopes

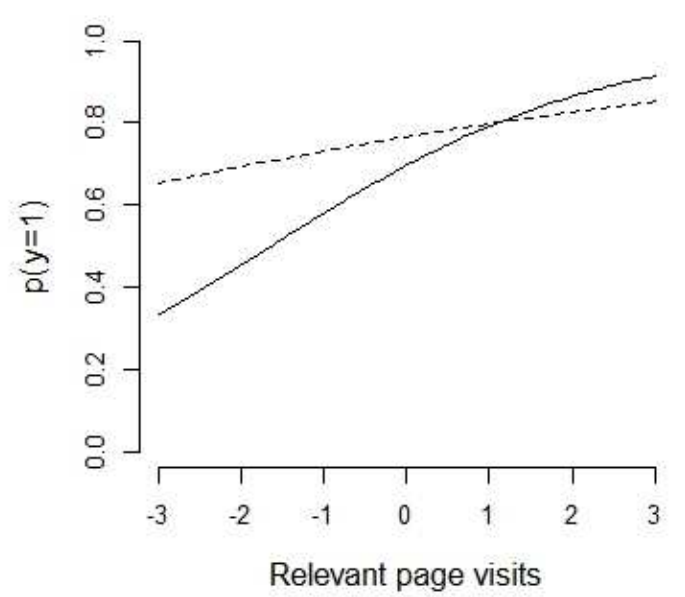

